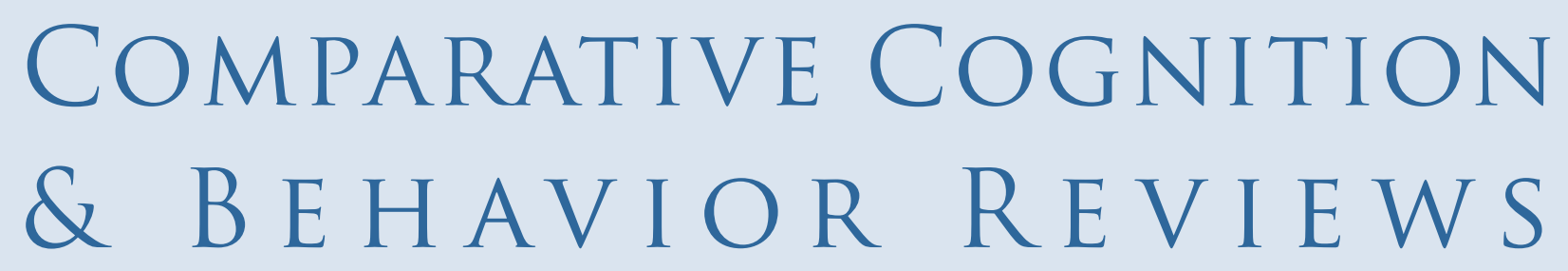

\title{
It's Hard to Be Social Alone: Cognitive Complexity as Transfer Within and Across Domains
}

\author{
Jennifer Vonk, Joy Vincent, and Victoria L. O'Connor \\ Oakland University
}

The field of comparative cognition is intent on demarcating cognition into social and physical domains; however, we argue that it is not useful to differentiate between cognitive processes involving social versus physical (nonsocial) phenomena. We argue that similar cognitive mechanisms underly reasoning about social and physical information and that it is transfer of knowledge and skills within and across these "domains" that is most informative with regard to identifying cognitive flexibility. We argue that social complexity, typically defined as group size, although important, has no special influence over the evolution of social cognition. Likewise, foraging complexity, typically conceptualized as dietary breadth, extractive foraging, and patchy food distribution, has no special influence over the evolution of physical cognition. Instead, researchers should treat social and foraging complexity as continuous rather than dichotomous variables that interact with one another and with environmental unpredictability to predict domain-general cognition that can be applied flexibly across novel features, contexts, and experiences. To answer long-standing questions about the selection pressures for cognition, researchers must consider species that differ in various aspects of their ecology on analogous tasks within and across domains.

Keywords: cognition, evolution, domains, social, foraging, flexibility, generalization

For the past 2 decades, comparative researchers have been working under the tacit assumption that cognition operates in two key domains: social and physical (Herrmann et al., 2010; Tomasello, 2000; Tomasello \& Call, 1997). Social cognition involves reasoning about social agents and mental states, whereas physical cognition involves reasoning about nonsocial phenomena such as time, space, and objects. Researchers are interested in how cognitive traits are phylogenetically distributed and shaped by an evolutionary history of adaptive challenges in social and physical domains. Yet there is little evidence that reasoning about social phenomena requires fundamentally distinct cognitive processes from those involved in reasoning about physical phenomena. There is also scant evidence that complex sociality is uniquely linked to skills in the social domain and that foraging challenges imbue organisms with cognitive skills that pertain strictly to the physical world. Although researchers refer variously to "cognitive complexity," "advanced," "higher order," and "sophisticated" cognitive processes as terms to capture the idea of general intelligence, it has also been a challenge to define and operationalize such constructs. We propose a shift from dichotomizing underlying cognitive mechanisms (and domains in which they operate) toward a focus on flexible transfer of knowledge and skill across domains. Whereas we argue for a more general approach to conceptualizing cognitive outcomes, we argue for a more specialized approach to quantifying the factors driving cognitive evolution.

Social and foraging complexity are commonly conceived of as independent predictors of cognitive complexity, whereas they must operate in concert. We confront the challenges of defining and operationalizing these three aspects of complexity and argue that selection pressures may best be examined in terms of specific continuous features rather than as dichotomous categories. For example, 
rather than labelling a species as social or asocial, it is important to consider various aspects of sociality including parenting and mating strategies and interactions with prey and predators. We use 'complexity' to refer to behaviors or cognitive representations involving a greater number of manipulations or relationships. For example, greater social complexity is exhibited when individuals must track a larger number of individuals in a dynamic environment or interpret a greater breadth of emotion states. Greater physical complexity may be indicated by a larger number of modifications to a physical environment or diet within a shorter time frame, or a larger number of manipulations to an object or travel route required to procure a food reward. These factors may interact and overlap. For example, more complex communicative skills may overlap with group size, territory size, or social structure. When examined in this way, it becomes clear that the assumed advantage for group-living species may undersell contributions from other socioecological pressures, such as weaning periods and foraging strategies.

A better understanding of the association between selection pressures and cognitive skills in different domains is hampered by the lack of a clear framework for assessing domain-general cognition across a range of species that differ as dramatically as, for example, spiders from chimpanzees. Given the impossibility of establishing standardized cognitive assessments applicable across such a range of species with such varied morphological adaptations, one option is to neglect the search for a general intelligence factor $(\mathrm{g})$ and to instead focus on specific traits (Vonk \& Edge, in press-a). Alternatively, it may be preferable to examine domain-general constructs such as flexibility that can be exhibited in different ways by different species (see also Colombo et al., 2021). For carnivores such as bears, flexibility may be shown in adjustments to diet across seasons (Vonk, in press). For orangutans, flexibility may be adjusting social organization based on predator threat, territory size, and captivity (Harrison \& Chivers, 2007). In general, flexibility can be defined as the ability to

Author Note: Jennifer Vonk, 654 Pioneer Drive, Oakland University, Rochester, MI 48309.

Correspondence concerning this article should be addressed to JenniferVonkatvonk@oakland.edu.

Acknowledgments: $X X X$ adaptively adjust behavior in response to socioecological changes or constraints. Thus, it includes the component of inhibiting previously rewarded but currently maladaptive behaviors, as well as the component of innovating new behaviors (Johnson-Ulrich et al., 2018). It should be expected that flexibility is related to complexity, as both constructs by definition involve breadth of phenomena and possible responses.

Although comparative psychologists traditionally focused on domain-specific traits and abilities, they have more recently engaged in the pursuit of a better understanding of general intelligence in nonhuman animals (hereafter nonhumans; e.g., Arden \& Adams, 2016; Bar-Hen-Schweiger \& Henik, 2020; Burkart et al., 2017; Damerius et al., 2019). Intelligence is almost always viewed through an anthropocentric lens (de Waal, 2016; Leslie, 2018) that fails to appreciate the diverse learning challenges, sensory modalities, brain structures, and cognitive mechanisms of nonprimate species. Bar-Hen-Schweiger and Henik (2020) presented a recent alternative that focuses on mental manipulations and noted that "the generalization of ability reflects learning, cognitive flexibility and improved adaptation" (p. 694). Similarly, we focus on the flexibility to apply cognitive processes across contexts - sometimes even crossing social and physical domains - as one hallmark of "cognitive complexity." For example, the human ability to apply empathy to inanimate objects may be seen as an extreme, but not necessarily adaptive, demonstration of flexibility, whereas a chimpanzee's ability to respond to competitive but not cooperative signals (Hare, 2001) would be a sign of inflexibility. Furthermore, the inability to interpret signs of emotion, such as fear, in species other than one's own would be a serious limitation and demonstration of inflexibility.

A lack of flexible responding can also be tied to an inability to generalize beyond specific perceptual features-for example, learning to expect the delivery of a food pellet upon hearing a tone at a particular frequency rather than expecting delivery of food pellets for relatively high- frequency but not relatively low-frequency tones. Researchers sometimes conflate this lack of generalization with associative learning. However, associative processes can explain generalization even when transfer stimuli are vastly different from training stimuli as long as they are more similar to the trained rewarded stimuli on some dimension than the unrewarded stimuli. In general, when examining transfer performance on experimental tasks, researchers have unnecessarily dichotomized associative processes from so-called "higher-order" cognitive processes. This is a false dichotomy, as these latter processes 
necessarily depend on the associative processes that are often demeaned by researchers as being characteristic of "lower" organisms (see also Vonk \& Edge, in press-b). Indeed, Macphail $(1983,1987)$ infamously argued that all of cognition could be reduced to associative processes. From here, he concluded that there are no fundamental differences in cognitive ability across the vertebrates-a position that was met with nothing short of derision at the time but is now viewed as prescient (Colombo et al., 2021). However, whereas Macphail's argument for similarity across vertebrates stemmed from a dismissal of the idea of so-called higher order cognitive processes, comparative psychologists embracing Macphail's "null hypothesis" today fall on the other side of the spectrum-instead seemingly motivated by a willingness to attribute "higher order" processes to a wider range of species.

Elsewhere, Povinelli and Vonk (2004) outlined what Povinelli (2020) now refers to as the Asymmetric Dependency Problem, in which "higher" processes necessarily depend on attention to observable phenomena, whereas processes involving reasoning about these observable features need not give rise to conceptual representations of the causal relations between them. That is, it is not possible to form "higher order" representations or abstractions of stimuli without first attending to their observable features. However, it is possible to reason about these observable features without forming more abstract relational interpretations of the stimuli. Thus, it is impossible to dissociate abstraction from associative processes, as the former depends on the latter. Any dichotomizing of cognitive processes (e.g., "higher" vs. "lower" associative processes) will be unproductive, as cognition is better conceived of as a complex network of associated processes, some of which are primarily implicit/automatic, canalized, and widely shared and others of which are possibly unique to particular species, with humans just one of many interesting examples. Here, instead of characterizing a particular type of process as being high level or low level, we indicate the extent to which learning (either behavior or knowledge) is applied broadly across features, contexts, and experiences, with the idea that less constrained generalization will be indicative of cognitive flexibility (as one form of intelligence). Thus, instead of focusing on whether transfer is based on observable features or an abstraction of the relation of the stimulus to other stimuli, we focus on whether learned behaviors or knowledge can be applied to novel scenarios (e.g., social vs. foraging), where other aspects of the problem change. To the extent that generalization extends beyond the confines of perceptual features (e.g., color or pitch) or combinations of features, we would considerate it a demonstration of cognitive flexibility-a hallmark of cognitive complexity.

An unfortunate extension of the inappropriate dichotomizing of associative and cognitive processes is the tendency to attribute associative processes to a behaviorist framework and to assume that a cognitive framework can elevate cognition beyond "simple associations." When researchers interpret the responses of nonhuman subjects with reference to the association of specific observable features or events with positive outcomes, their interpretations are described as behaviorist. In contrast, when they appeal to constructs such as theory of mind, metacognition, or abstract causal reasoning, their interpretations are said to be mentalistic or cognitive. For behaviorist accounts, all responses are grounded in previous experience and learned associations between behaviors and outcomes, whereas cognitive accounts allow for individuals to make inferences about outcomes they have not yet experienced and to predict outcomes based on unobservable causal forces such as mental states. This long-standing tension between behaviorists and cognitivists represents another unnecessary dichotomy that is poorly grounded in our current understanding of cognition, which recognizes the important role of both behavioral inputs and the cognitive processes that act upon them. Povinelli's (2020) Asymmetrical Dependence problem is relevant here again to remind the reader that one cannot make inferences about unobservable causal forces without first attending to the observable behaviors from which internal states can be inferred. Importantly, unobservable cognitive mechanisms cannot be inferred in the absence of associations between observable manifestations. Furthermore, one need not embrace behaviorist principles exclusively to appreciate that an animal's ecology informs its responses and abilities (Leslie, 2018). Even apparently complex behavior, such as tool use, depends on both innate predispositions and learning, including both operant conditioning and social learning (Amodio et al., 2018). Rather than being bogged down in debating the supremacy of behaviorist or cognitive accounts of leaning, we advocate for a focus on the generality versus specificity of learning that occurs.

Researchers have sometimes contrasted "rulebased" responding with associative learning, where rules are assumed to represent more abstract representations of the relations between stimuli/behaviors/events (Povinelli, 2020). Rather than fixating on whether an association between observable features (or between functional features and outcomes) or an abstract representation of 
unobservable causal forces defines learning, we focus on how broadly knowledge is applied once acquired. Whereas rules referring to the relations between behaviors and outcomes are more likely to be flexibly and broadly applied compared to associations between specific perceptual features or between perceptual features and outcomes, rules can also be limited in the extent to which they are generalized beyond the learning context. Moreover, there is evidence that animals, such as pigeons, that do not exhibit rule formation in categorization tasks may nonetheless exhibit a great degree of flexibility within the context of an associative learning system (O'Donoghue et al., 2020). Focusing on the breadth of transfer across tasks, contexts, and features of the environment also eliminates the need to focus on analogous tests of comparable abilities in species that vary considerably in morphological adaptations.

Although we have thus far focused on breadth of generalization as indicative of cognitive flexibility, we also recognize that overgeneralization would conversely be indicative of cognitive limitations. For example, researchers have championed the flexibility of ants that seem to distinguish between conspecifics and alien species in their burial practices (Renucci et al., 2010). However, in the case of ants that remove live conspecifics doused with a chemical typically associated with death (Diez et al., 2013), the ants' response is hardwired and restrictive, exhibiting little to no flexibility. Moreover, this response might be classified as a social behavior, but the ants are responding in a fixed manner to a physical compound rather than reasoning about whether conspecifics are alive or dead, highlighting the futility of labeling the cognitive mechanism as social or physical in nature.

We hope to contribute to a clearer understanding of how cognitive flexibility evolved across social and physical domains. Currently, it is unclear how species' socioecologies prepare them to learn-for example, whether a complex social environment confers greater natural social skills through inheritance or provides the foundation from which animals might acquire skills and concepts that they are not innately endowed with (or some combination of the two mechanisms). We argue that the distinction between social and physical cognition has been oversold and that lack of transfer across domains indicates a cognitive limitation rather than domain specificity per se. Last, following Sol (2009a, 2009b), we argue that the variability and unpredictability of the environment (including social and foraging complexity), rather than a single ecological factor, predict breadth of transfer across domains.

\section{Hypotheses Regarding the Evolution of Cognitive Complexity}

Various hypotheses have presented possible selection pressures for cognitive evolution. For example, the Machiavellian intelligence (Byrne \& Whiten, 1988) and social brain hypotheses (Dunbar, 1998, 2014; Humphrey, 1976; Jolly, 1966) argue that larger brains evolved to deal with social challenges. These hypotheses center on the observation that group-living organisms depend on the ability to evaluate, remember, and generalize from observed group dynamics to achieve reproductive success. Cognitive abilities that may be selected for in complex societies include self-awareness, perspective taking, reputation formation, empathy, social learning, and culture (Hauser, 1988; Whiten \& van Schaik, 2007). Complex social relationships presumably select for "more effective strategies of achieving social success (including deception, manipulation, alliance formation, exploitation of the expertise of others, etc.)" (Gavrilets \& Vose, 2006, p. 16823). Larger brains are capable of supporting this greater cognitive complexity.

Social success is facilitated by the smaller litter sizes, longer weaning periods, and greater opportunities for social learning that typically occur in group contexts. With the added protection that groups provide, parents can invest more in fewer offspring and for longer. This reproductive strategy allows for the investment in larger brains; the longer protective period of infancy provides the brain time to develop compared with species that do not invest in significant offspring rearing. In addition, group living offers the benefits of social learning, which in turn may facilitate the development of a greater breadth of solutions to problems (e.g., Hauser, 1988), which may be conceptualized as flexibility. There is less need to learn via trial and error or to innovate (i.e., develop a novel solution to a problem) when one can hijack another's expertise.

The cultural intelligence hypothesis (Whiten \& van Schaik, 2007) emphasizes the role of social learning in the evolution of intelligence; however, the authors speculate that both innovation and social learning encourage success of species in new environments, which encourages evolution (Whiten \& van Schaik, 2007). Although much has been made of the potential importance of sociality, it is unclear whether sociality selects for domain-general skills, or specifically for social cognitive skills, such as social learning. In our view, much work needs to be done to identify whether factors selecting for cognition do so with regard to domain-general or domain-specific cognitive skills. For example, social living may contribute to 
skills such as theory of mind and reading of communicative cues but may have little influence over the emergence of domain-general abilities such as causal reasoning and working memory. Furthermore, if group members depend less on innovation compared with solitary individuals, it is possible that innovation and flexibility are more strongly selected for in solitary rather than group-living individuals. Given that innovation may be a central component of cognitive flexibility, we argue for a greater emphasis on nonsocial selection pressures, such as dietary breadth and unpredictability of physical environments (Sol, 2009).

As one example of a hypothesis focused on foraging factors, the technical intelligence hypothesis (Byrne, 1997) emphasizes the need to extract food sources as a driving force for cognition. Specifically, consuming foods that require tool use or manipulation to extract should be linked to a better understanding of object properties and relations, enabling efficient tool use and possibly causal reasoning. Similar to our question presented earlier as to whether social complexity should lead to domain-specific or domain-general skills, it is unclear whether selection pressures such as the need to extract food operate on specific cognitive skills or lead to domain-general skills such as the capacity for inhibition and innovation, relational understanding, and causal reasoning. Causal reasoning itself is a nebulous and difficult construct to define and, indeed, has been argued to have little utility in clarifying the cognitive mechanism underlying animals' behaviors (Povinelli \& Henley, 2020).

Although most commonly considered separately, social and foraging challenges need not be viewed as mutually exclusive predictors of cognition. Indeed, the technical intelligence hypothesis recognizes that group living may facilitate the transfer of knowledge regarding tool use and other skills necessary for foraging success. Hypotheses centered on foraging complexity (e.g., Milton, 1981) rarely contrast predator and prey species or view hunting as a primary driver of cognition. However, hypotheses centered on group living have acknowledged the role of collaborative hunting in potentially shaping cooperation and other social skills (Boesch, 2003). For example, Strübin et al. (2011) presented a fascinating argument that collaborative hunting may select for group living rather than treating group living as the selection pressure for presumed sophisticated cognitive abilities underlying collaboration. Their hypothesis was based on observations of collaborative hunting in goatfish (Mullidae) that either adopted solitary lifestyles or formed small groups. Group members engaged in collaborative hunting, taking on the role of chasers or blockers, whereas solitary fish engaged in a different foraging technique-exploiting stationary hidden prey. Notably, the authors found it unnecessary to refer to complex cognitive processes to explain the flexibility exhibited by the hunters; their behavior could be explained solely by the rule "Maintain maximum distance from conspecifics." Other observations support the interplay of foraging and social factors in determining cognition. Even relatively solitary species may have long weaning periods that confer the opportunity for young to learn socially from their mothers (e.g., bears; Mazur \& Seher, 2008; Morehouse et al., 2016). Next, we expand on the need to consider sociality and foraging complexity in a continuous rather than a dichotomous manner and to recognize that these broad classes of selection pressure are not mutually exclusive.

The cognitive buffer hypothesis (Sol, 2009a, 2009b) posits that increased brain size evolved in response to increasing variability in the environment. This is particularly salient for species with a generalist ecology, as they are likely to face various novel or unfamiliar conditions that require increased behavioral flexibility (Sayol et al., 2016). Although defined variously (Audet \& Lefebvre, 2017), behavioral flexibility is typically thought to consist of inhibition as well as innovation; however, it is not always clear how these components are related (Johnson-Ulrich et al., 2018). Both components are important because to behave flexibly, individuals must learn to cease engaging in behaviors that are no longer productive and to initiate novel responses that may have no previous history with reward. Thus, individuals may have to generate novel solutions through insight and trial and error rather than being able to generalize from previous learning histories. Recently, behavioral flexibility has been proposed as a proxy for general intelligence and a promising complement to more traditional measures of learning and executive function (Reader \& MacDonald, 2003). Understanding differences in cognitive and behavioral flexibility across taxa will be aided by appreciating the diverse social and ecological challenges faced by different species. Dynamic environments likely require more innovation than unchanging environments (Sol, 2009a). According to the cognitive buffer hypothesis, the primary function of a large brain is to "buffer" against environmental hurdles and increase survival rates (Allman et al., 1993; Deaner et al., 2003). However, complexity is determined by both dietary and social factors. A large brain can facilitate cognitive processes such as innovation through the construction of novel or altered behaviors (Sol, 2009b). Socially mediated learning allows the transmission of innovations to offspring and/or group members. Evolution of innovative 
behaviors may favor large social systems that present more opportunities for close kin to benefit from the innovations over generations. The ability to learn socially may lead to increased knowledge and innovation over time, increasing the cognitive skills of younger generations.

Of interest, Osiurak and Reynaud (2020) recently outlined a technical reasoning hypothesis that turns traditional thinking about cumulative technological culture on its ear. These authors eschew the distinction between social and asocial learning mechanisms (e.g., Boyd et al., 2011; Dean et al., 2012; Hernik \& Csibra, 2009; Tennie et al., 2009; Tomasello, 1999) and emphasize the role of nonsocial cognitive processes as necessary for cumulative technological culture. In their theory, technical reasoning skills also underlie metacognition and theory of mindcognitive abilities that have previously been examined as facets of social cognition. However, they reject the notion of technical reasoning as a domain-general skill, instead defining it as the ability to reason about physical object properties. They do note that technical reasoning is both analogical and causal; that is, one sees the relevance of knowledge from one situation to another and also understands the effects of the knowledge on the environment. However, they are careful to acknowledge that the transmission and extension of knowledge does not necessarily depend on a causal understanding of the mechanics of the technology. We diverge from Osiurak and Reynaud in that we see the ability to reason about causal and unobservable properties and generalize their application across contexts and experiences as a domain-general skill. We see no reason why knowledge gained in a physical context cannot be transferred to a social context (see Bastos \& Taylor, 2020). For instance, although controversial, some have advanced the idea that individuals use conspecifics as "social tools" (e.g., begging an individual that can retrieve an out-of-reach object and pass it to the solicitor) much like they manipulate physical tools (e.g., using a stick to rake in an out-of-reach food item; Bard, 1990). We do not distinguish between physical and semantic knowledge here. These differences notwithstanding, there is significant agreement on the notion that the ability to adapt and innovate is central to cognitive achievement. In a 1963 article in Southwestern Social Sciences Quarterly, Leon C. Megginson paraphrased Darwin in this manner:

According to Darwin's Origin of Species, it is not the most intellectual of the species that survives; it is not the strongest that survives; but the species that survives is the one that is able best to adapt and adjust to the changing environment in which it finds itself. (p. 4)

\section{Defining Complexity}

\section{Foraging Complexity}

Before we can evaluate the contributions of social and foraging challenges to shaping cognition, we must consider how social and foraging complexity should be defined. There has been greater effort toward building a consensus of what constitutes foraging complexity (relative to social complexity), with researchers stressing the extent to which organisms adopt a generalist diet or exhibit flexibility in foraging strategies; need to extract food such as nuts, fruits with hard or spiny outer shells, insects that burrow in trees or underground, and so on; and exploit food resources that are patchily distributed in time and space (Byrne, 1997; Milton, 1981). Animals that depend on a single food source that may be acquired using a single behavioral strategy, such as anteaters, are likely to be less cognitively flexible compared with omnivores that eat a variety of foods requiring different behavioral techniques to acquire. For example, within the bear family, several omnivores, such as black bears and brown bears, eat fish and other meat, nuts, berries, seeds, and so on. Others, such as polar bears, are obligate carnivores and are expert predators of seals and other aquatic animals. Giant pandas subsist entirely on bamboo and have developed specialized adaptations for stripping and consuming bamboo. Thus, there should be a range of cognitive complexity within the ursid family if dietary factors predict cognition (Vonk, in press; Vonk et al., 2020) with brown bears-for example, outperforming polar bears and giant pandas on measures of general cognition because brown bears are generalist foragers. Although evidence exists that brown bears have the largest relative brain size among ursids (Gittleman, 1986), which may be linked to their generalist diet, data on cognition across bear species are too sparse to answer questions about foraging complexity and cognition in this family to date (Vonk, in press).

If extractive foraging is key to cognitive complexity, one might not expect cognitive differences between these bear species, because extraction is involved in procuring bamboo for giant pandas and seal meat for polar bears (arguably) as well as items in the diet of brown bears. Furthermore, hunting live prey is likely to require a cognitive skill set not needed, and therefore not selected for, in pandas. Cognitive differences between bears could help shed light on the factors that have been critical in shaping those differences, although it is important to consider that it is unlikely to be a single factor that explains differences in all traits. For example, specialists such as giant pandas 
might outperform other species on tasks that require specific skills, such as certain spatial memory tasks. Tarou (2004) found that pandas outperformed spectacled bears on a win-stay foraging task, whereas the opposite was true of a win-shift task. In win-stay strategies, animals search again at sites where they have previously found food; in win-shift strategies, animals do not return to previously depleted sites. Thus, use of these strategies should depend on whether animals are foraging for foods that replenish at short intervals - in other words, their "patchy distribution" in time and space. In another study, pandas outperformed Asian small-clawed otters in tests of working and reference memory (Perdue et al., 2011). These otters exhibit more seasonal flexibility in their diet compared with pandas, consume foods such as shellfish that require extraction, and are much more social compared with pandas. Therefore, in tests of general cognition we would expect small-clawed otters to score better than pandas. However, given that pandas consume plants that replenish, it is not surprising that pandas may perform better than otters on win-stay spatial memory tasks. Thus, it is not sensible to focus solely on, for example, social complexity or extractive foraging as predictors of cognition without considering the particular cognitive skill being tested and the animal's ecology in its entirety.

\section{Social Complexity}

Despite the greater focus on sociality as a predictor of cognition, there has been less agreement as to what constitutes complex sociality, although a recent special issue was devoted to the topic (Kappeler et al., 2019). Typically, researchers forge a sharp divide between social and "asocial" species, glossing over the great variability in more specific elements of sociality. For instance, sociality is commonly defined as network size (Dunbar \& Shultz, 2007; Freeberg et al., 2012), which varies quite considerably, or as the structure of the society itself, including whether it contains smaller groups in a fission-fusion dynamic (Kappeler et al., 2019).

Sociality should not be restricted to group-living species, because that would exclude other types of social relationships such as those between bonded mates, parents and offspring, and communal breeders (Boucherie et al., 2019; Kappeler et al., 2019). We advocate for Kappeler and colleagues' (2019) framework of social organization, social structure, mating system, and care system as distinct components of social complexity. However, even animals that have traditionally been viewed as asocial, such as orangutans, brown and black bears, and clouded leopards, exhibit social behavior and recognize rank when they encounter conspecifics. Recently, Elbroch and colleagues (2017) found that pumas - considered a solitary species - tracked social interactions of neighboring pumas and exhibited reciprocity in tolerance of conspecifics. This finding is the first indication of a social strategy in a solitary species and demonstrates a greater degree of sociality in these animals than previously recognized (Vonk, 2018). Species that share a prolonged weaning period with their offspring should be distinguished from species that, for example, bury their eggs and never have contact with their offspring, such as sea turtles (Kamel \& Mrosovsky, 2005). That is, socialization that occurs before weaning should be considered to contribute to the development of an organism's social cognition. In primates, we have argued that reproductive strategies may be especially critical to shaping cognition (Vonk \& Edge, in press-a), and indeed pair bonding has emerged as the primary predictor of large brains in ungulates and birds (Dunbar \& Shultz, 2007; Shultz \& Dunbar, 2007). Thus, we propose extending Kappeler et al.'s framework to account for social interactions that take place outside the context of the group.

Additionally, although Lott (1991) highlighted the importance of intraspecific variation in aspects of social complexity 3 decades ago, sociality is generally attributed at the species rather than the individual level. Given our focus on laboratory studies as well as field studies, we cannot ignore the importance of an individual's social experiences and rearing history, and its possible impact on cognitive performance (Boesch, 2020). For example, it is possible for an individual to belong to a large social network but have few individual contacts. The current structure of the social group may lead to intraspecific variation in behaviors such as feeding tolerance (DeTroy et al., 2021). Additionally, an individual's network may appear quite different depending on the analysis that is used to identify social networks (Castles et al., 2014). Individuals that lack typical socialization during critical periods of development should not be classified as social solely with relation to species-level sociality. Similarly, individuals that have not been exposed to typical physical environments might not exhibit species-typical abilities. For example, it is known that impoverished environments can impact spatial memory in rodents (Rosenzweig \& Bennett, 1996). Thus, in determining the inputs to an animal's social environment, both individual and group-level experiences must be considered (DeTroy et al., 2021). In what follows, we discuss particular types of flexibility and generalization that can occur and that might be impacted by these environmental factors. 


\section{Generalizing Across Features of the Stimulus or Environment}

Tool use was once considered a unique and defining trait of humans (reviewed in Striedter, 2013) - a notion that was overturned by observations of termite fishing in human's closest extant relative, the chimpanzee (Goodall, 1964). The comparative record was then updated to suggest that tool use was exhibited by a few of human's closest relatives. For example, chimpanzees and capuchins are frequent spontaneous tool users in the wild and in captivity. It is now widely recognized that many species exhibit some form of tool use (reviewed in Bentley-Condit \& Smith, 2009) including dolphins (Patterson \& Mann, 2011), elephants (Hart et al., 2001), bears (Deecke, 2012; Waroff et al., 2017), chimpanzees (Pruetz \& Bertolani, 2007), and some other nonhuman primates (Visalberghi $\&$ Trinca, 1989). Most recently, researchers have focused on tool use in avian species, such as corvids (Teschke \& Tebbich, 2011), finches (Teschke et al., 2011), and parrots (van Horik \& Emery, 2016). Thus, the current interest in nonhuman tool use has moved beyond determining its ubiquity across species and is focused on characterizing species' differing cognitive representations of relations between tools and other objects.

Animal tool use studies provide a prime example of the challenges that emerge when one attempts to contrast competing cognitive mechanisms as explanations for the observed behavior, reliance on perceptual features alone versus a deeper understanding of tool and object properties, and the physical laws that govern their association inferred from attention to relations between those perceptual features and outcomes. By definition, tool use requires an individual to manipulate two different objects outside their bodies to meet some end (Tebbich et al., 2007). This simplified definition focuses on the physical behaviors associated with tool use but fails to address the more nuanced cognitive processes needed to effectively use tools. There has been a growing shift in literature away from "success testing," which dichotomizes performance into two exclusive results - success or failure (Taylor, 2014). This shift allows for a greater focus on the underlying cognitive mechanisms at play, providing a more comprehensive and nuanced understanding of tool use and the conditions under which this behavior has evolved. This research effort, however, continues to be stymied by diverging interpretations of the results-namely, whether tool use can be explained as a manifestation of causal reasoning or more simplistic associations that result in the appearance of causal reasoning (Teschke et al., 2011). As
Shettleworth (2010) cogently argued,

There is no good evidence that anything other than the mechanisms of associative instrumental learning . . . underlies tool using by any nonhuman species. ... [But] some animals seem quite good at recognizing the functional relevance of tools, and this could reflect a predisposition to perceive the affordances of certain classes of objects. (p. 412)

As just discussed, it is an empirical challenge to differentiate between tool use that (a) results from associations between perceptual properties and outcomes and (b) is demonstrative of complex and abstract conceptual representations of object relations. These more complex processes have been notoriously difficult to define but are most often conceptualized as "causal reasoning" or "causal understanding"- terms used to describe the ability to both understand the physical forces on a causal level and subsequently formulate and generalize rules to novel situations based on this understanding. Seed and Byrne (2010) postulated that "low-level" cognitive functions relied on attention to superficial, observable features (i.e., pull from the side closest to the food), whereas "high-level" functions encompassed mental representations of the underlying physical properties of a task (i.e., gravity, surface continuity, weight, opacity).

Determining the mechanism through which animals exhibit tool use is made more complicated when attempting to parse out the application of complex rules from attention to perceptual features of a task. Is it that an individual has generalized a rule about surface continuity, understanding the causal properties of the task (i.e., a reward cannot pass over a noncontinuous surface because gravity will cause the reward to fall down a hole), or did they simply form an association between features and outcomes (i.e., a noncontinuous surface means they will not receive a reward)? Povinelli and Henley (2020) argued that because higher order representations of physical forces must be predicated on the observation of perceptible object properties and relations, there is no experimental design that would allow researchers to draw conclusions about higher order processes with tool use. In any procedure, the individual will be presented with an object that has observable relevant functional features, even if the specific object is novel to them. There is no way for the subject to extract a relevant conceptual rule from the situation if this is not the case. Therefore, when first-order reasoning about a physical problem is both sufficient and necessary to find a solution, it is imprudent to attempt to explain the pattern of behaviors through higher order processes. Despite the consequent pessimism regarding the ability of any 
experiment to identify the existence of these higher order processes in nonhumans, current experimental paradigms have the ability to indicate the specific features to which nonhumans attend when generalizing the use of familiar objects to novel objects and contexts.

Although unable to clearly disentangle representations of causal forces from representations of perceptual features, researchers can still answer many interesting questions concerning the nature and development of nonhumans' understanding of the properties of objects. One question concerns the role of learning mechanisms such as trial-and-error learning versus insight, although the concept of insight itself is controversial (Striedter, 2013). Köhler (1925/2019) defined insight as the awareness of functional relationships in a given situation and its rapid application to formulate a solution to the present situation. Köhler's work with chimpanzees was groundbreaking and opened the floodgates for future investigations into insight in other nonhuman species. One notable study presented the chimpanzees with a suspended banana that was out of reach; the arena also contained a number of boxes and sticks that could be used to reach the fruit. Sultan was the most adept at solving the given problem, learning in less than a day to stack the boxes under the banana to reach it. Although Sultan was unable to immediately solve the problem, requiring multiple attempts to stack the boxes in such a way that they would not topple over, the speed at which he was able to ascertain a solution to the problem was remarkable. Köhler described the "moment of insight" as, after a period of thinking about the problem and possible solutions, the chimps rapidly began to use the tools at their disposal-namely, the boxes - to reach the banana. The results of this and other similar studies conducted by Köhler have been criticized for these interpretations, with many suggesting that the chimpanzees' behaviors are more in line with trial-and-error-style problem-solving rather than insight (Chance, 1960). This critique has been further supported by the high level of familiarity that the chimps had with the tools at their disposal. They had time to become familiarized with the structure and functionality of the boxes and sticks, thus shedding doubt on the reported rapidity with which Sultan appeared to have solved the box-stacking problem - as even with previous access to the boxes, he still was unable to complete the task immediately (Chance, 1960).

In a similar paradigm, Epstein and colleagues (1984) found that, in response to a banana suspended out of reach, pigeons moved a box from its initial location to directly under the banana, allowing them to access it. Despite the similar behaviors demonstrated by the pigeons, the researchers argued that this was the result of stringing together a series of previously conditioned behaviors rather than an example of "insight." Similar critiques have been lofted at the interpretations of Köhler's studies; Köhler was able to objectively demonstrate a series of behaviors that resulted in a solution but was only able to speculate about the underlying cognitive processes. Fuller (1990) argued that insight, as defined by Köhler, is not predicated on a deep conceptual understanding of the physical properties involved but rather is a reflection of the convergence of perceptual and experiential cues. Köhler likely made a false attribution to higher order processes, when the most parsimonious (and sufficient) explanation required postulating only first-order relational reasoning.

The principle of parsimony is paramount in the discussion of tool use; it is prudent to default to the lowest denominator of cognitive complexity that can explain a pattern of behavior (Teschke et al., 2013). However, one should be careful not to equate the idea of "lower level" complexity with the idea of inferiority or simplicity. Instead, one should focus on whether a particular level of processing is dependent upon an earlier level. For example, even humans cannot infer causal forces such as gravity without processing observable cues such as falling objects. Thus, predicting outcomes based on observable cues reflect only lower level processes. In testing procedures, immediate transfer of tool use from a familiar to a novel context has been taken to support the notion that the subjects abstracted a generalized rule relating to the causal relationship between their tool usage and the resulting reward (Striedter, 2013; however, see Barker \& Povinelli, 2019, for a discussion of why even Trial 1 performance cannot be taken as diagnostic of causal representations). In contrast, the inability to immediately transfer the use of a tool to a novel context is often taken to suggest that the individual attended to a specific element of the task, rather than abstracting a general rule about the physical properties involved (van Horik \& Emery, 2016). For example, if an individual uses the weight of an object (regardless of whether this is felt tension when lifting or an attribution of weight as a feature of an object; Povinelli, 2012) to topple a structure, knock a coconut from a tree, or break into a nut, one might expect them to generalize that a more heavily weighted object is more apt to aid in solving the so-called Aesop's Fable task compared with a lighter object. In this task, subjects throw stones into a tube filled with water to gain access to a now-floating reward. For our purposes, it is less relevant to what feature the subjects attended (proprioceptive feedback or object weight), and more important that the knowledge gained by solving one problem 
could be used to solve another, in a different context and across different task features. Rooks (Bird \& Emery, 2009) and crows (von Bayern et al., 2009) succeeded in the Aesop's Fable task. The rooks had the opportunity to observe stones being put into the tube, whereas the crows had no such prior experience. Both species generalized from the features of the task - the rooks from their opportunity to observe the task directly and the crows from prior experience with different, but conceptually similar items, which may represent a broader form of transfer. It is unlikely that an individual would come into a testing situation entirely naïve to previous associations related to physical properties; thus even the crows' impressive transfer was based on observable features of their environment.

It is important for researchers to recognize and understand the experiences from which their test subjects may be generalizing, especially when it comes to interpreting the results of studies, so as to not overstate their findings. In one study championed as evidence of causal reasoning, apes were presented with two ropes, and each was affixed to a bucket-one with food and the other without (Schrauf \& Call, 2011). The bucket with food was heavier and thus harder to pull. The apes demonstrated a preference for the heavier bucket, suggesting that they understood something about the relationship between the increased weight of the bucket and the presence of food in the bucket. This preference was lost, however, when they were presented instead with arbitrary cues about the location of the food, using colors to differentiate between the two buckets. This suggests that the functional cues of weight were more salient in solving the task relative to the (in this case) arbitrary color cues. It is tempting to attribute these results to the ability to understand and generalize physical properties on a causal level, but there is a more parsimonious explanation. It may not be that the individuals formed an abstract concept about weight and its causal significance but rather that they generated a simple association between the tension upon lifting and a reward. It makes sense for apes to attend to cues related to the weight of a branch in determining if it will yield food, given their semiarboreal nature. Although color is also a relevant cue to ripeness of fruit, for example, it would not be linked to the presence or absence of hidden food. Animals should be predisposed to form strong associations to ecologically pertinent forms of information in relevant contexts, even if they do not abstract causal relations between the notion of weight and gravity.

It is not simply this rule of parsimony but also a general lack of supporting evidence that calls into question the link between tool use and higher order physical cognition. Woodpecker finches are habitual tool users; however, their tool use in the wild appears to be dependent on habitat and resource availability (Tebbich et al., 2002). This provides researchers with the opportunity to compare the performance of tool-using and non-tool-using individuals of the same species. Teschke and colleagues (2011) compared tool-using and non-tool-using woodpecker species with small tree finches-a closely related, non-tool-using species - on a battery of physical tasks. These tasks included a simple reversal task to test their general learning abilities; wherein after an acquisition phase, during which they learned to associate a color with a reward, the color-reward pairing was changed in the reversal phase. The second task was a non-tool-using physical task, called the seesaw task, that was designed to test the subjects' understanding of surface continuity. They had to perch on one of two levers that would cause the reward to roll down the surface toward them; however, one side of the seesaw surface was continuous, and the other had a hole, resulting in the subject failing to access the reward if they chose the incorrect lever. In the third task, the subject had to select one of two canes, only one of which would result in the retrieval of a reward. The final task was a novel box-opening task designed to test their perseverance and ability to apply a series of behaviors. The presupposition was that, if tool use evolved in conjunction with enhanced cognitive abilities, the woodpecker finches would be more successful than the small tree finches. In contrast, small tree finches outperformed the woodpecker finches on the physical tasks, making fewest overall errors on both the seesaw task and the cane task; however, this difference was significant only when compared with the non-tool-using woodpecker finches. The woodpecker finches outperformed the small tree finches only on the novel box-opening task, which the researchers postulated may be attributable to their extractive foraging ecology, which may result in increased perseverance, a trait necessary in opening the box (Teschke et al., 2011).

A similar study comparing woodpecker finches' and small tree finches' performances on a two-trap tube task yielded similar results, contradicting the narrative of the coevolution of tool use and advanced physical cognition (Teschke \& Tebbich, 2011). The trap tube task requires individuals to use a tool to gain access to a reward that is inside a horizontal clear tube; they must avoid pushing the reward into the vertical trap located along the bottom length of the tube. Successful reward procurement can be accomplished through following rules, such as "push from the side farthest from the food" or through understanding observable features such as surface continuity 
and unobservable causal forces such as gravity. Teschke and Tebbich (2011) found that the two species performed similarly on the task, suggesting that physical cognition may have evolved in a context independent of tool use. Contrary to these results, however, when comparing tool-using New Caledonian crows with non-tool-using carrion crows (Teschke et al., 2013), the expected pattern of tool-using individuals outperforming non-tool-using individuals on physical tasks emerged. It is, thus, unclear how experience with tool use impacts performance on a variety of tasks. Neither species of crows are highly social corvids compared to, for example, the Florida scrub jay (Clayton \& Emery, 2007; Holzhaider et al., 2011), again suggesting that aspects of their foraging ecology, such as their generalist diet, may be more pertinent to their high level of cognitive skill.

If tool use generally reflects greater cognitive complexity, this should be evidenced by a relationship between tool use and other markers of general cognitive ability. Some of the most compelling evidence in support of a relationship between complex cognition and tool use are the strong correlations between brain size and tool use in primates (Reader \& Laland, 2002) and birds (Lefebvre et al., 2002), as well as higher degrees of folding observed in the cerebellum of tool-using birds compared with their non-tool-using counterparts (Iwaniuk et al., 2009). This presumed relation between brain size and cognitive complexity has been strongly supported, given that larger and more complex brains may be predictors for enhanced rule formation ability (Teschke \& Tebbich, 2011). Although tool use has been heralded as a clear indication of advanced physical cognition (Emery \& Clayton, 2009), there is scant evidence to support this claim, with most studies concluding that complex cognitive abilities may not be a necessary precursor to tool use (Teschke et al., 2011). The underlying argument contesting the complexity of tool use in animals is that such behaviors may be more representative of widely shared learning mechanisms rather than cognitive complexity.

A more fruitful line of inquiry examines other possible selection pressures driving tool use. Such studies have suggested the possibility that tool use may be driven by environmental or social factors, indicating the need for pointed investigations into ecologically relevant forces that may shape the development of tool use in a variety of species. Various conditions in the wild may contribute to a species' ability to use tools, either through abstractions of perceptual features or unobservable causal forces (note that in both cases the animal is assumed capable of abstracting rules or representations, but what differentiates the mechanism is the nature of the underlying representation). It may be advantageous for species that commonly encounter variations of the same problem to formulate and generalize a rule to such situations. Macaws, which live in forest canopies and feed off berries and seeds, would benefit from understanding distal object relations, dictating how and which objects they can pull toward themselves to increase their foraging success (van Horik \& Emery, 2016). The adversities faced by animals in the wild select for cognitive mechanisms that allow individuals to be successful in experimental tasks. Woodpecker finches, for example, are extractive foragers, which may explain why they were particularly adept at a novel box-opening task. Similar to the opaque box they were presented with in the experiment, when foraging in the wild, they are unable to have visual contact with their reward, so they may be biologically prepared to excel at this type of task (Teschke et al., 2011). Many species that have never exhibited tool use in the wild have demonstrated the ability to spontaneously use tools in experimental tasks (Spaulding \& Hauser, 2005), stressing the importance of current environmental inputs over natural ecologies in some contexts. In sum, research efforts should focus less on the perceived dichotomy between simple associations and causal reasoning about physical properties underlying tool use in nonhuman animals and more on how their ecological and evolutionary histories contribute to this capability (see also Povinelli \& Henley, 2020).

Although we have focused on attention to observable features in the context of physical tool use, many social behaviors can be attributed to generalization that occurs from specific features as well. For example, domestic piglets exhibited the ability to ascertain the identity of handlers through an associative process (Brajon et al., 2015). They demonstrated differential responses to novel handlers with whom they were unfamiliar based on a combination of their experiences with previous handlers and the behaviors of the novel handlers. If their first interaction with a handler was positive, it resulted in an overall decrease in aversion to unfamiliar handlers, particularly those that were motionless, in comparison with those slowly moving toward the piglets. The pigs appeared to associate unfamiliar handlers that were motionless with more positivity, showing increased approach and exploratory behaviors, compared with the unfamiliar handlers that were moving. This suggests that the piglets were able to both discriminate between handlers and formulate rules based on the context of their social environment and observable cues such as speed of motion. If pigs could be shown to use motion outside of the context of approach/avoidance of handlers 
to make inferences about which humans were most likely to provide food or assistance to other individuals, it would constitute evidence that they generalized from their own experience with the handlers to a general characteristic (or reputation; see next) that would transfer to the handler's behavior with other pigs, and perhaps humans as well.

\section{Generalizing Across Contexts}

The ability to generalize knowledge or apply a behavior established in one context to a novel context indicates cognitive flexibility. In the social context, flexibility might be exhibited if animals generalize from avoiding food that has been gazed upon by dominant animals in a competitive context (Hare et al., 2000) to understanding that cooperative humans must direct their gaze toward the object of their desire in a cooperative instrumental helping task (Warneken \& Tomasello, 2006). ${ }^{1}$ However, researchers have instead typically argued that even chimpanzees should exhibit these attention reading skills only in the contexts that are closest to the conditions under which they have evolved - that is, competition (Hare, 2001; Hare \& Tomasello, 2004). When transfer occurs within the same narrow constraints, it is likely not indicative of the generalization of an abstract rule but simply the generalization from learning about some observable features to the most physically similar available option. For example, researchers often proclaim evidence for causal reasoning when animals generalize use of one kind of rigid tool (like a stick) to another (a tool made of PVC) while avoiding use of more pliable objects (ferns, rubber strips), citing the fact that a novel tool was not specifically associated with food rewards in training. However, generalization based on perceptual similarity is widespread within the animal kingdom and is a fundamental aspect of learning. Using a salient feature such as rigidity to choose between two or more tools is a kind of generalization based on features that is unlikely to differentiate animals of differing levels of cognitive complexity. Nevertheless, even in the case of such readily observable cues, chimpanzees sometimes fail to explore or test such features before attempting to use tools in experimental tasks (Povinelli \& Frey, 2016) and sometimes fail to demonstrate transfer between functionally similar tasks (Martin-Ordas et al., 2008).

Although we focused on a form of physical cognition-tool use-in the previous section, perceptual features can also be used to generate successful performance on tasks of social cognition. For example, chimpanzees

1. Note that neither instance requires an understanding of "seeing" as an unobservable mental state. can use physical behaviors such as transfer of food by hand within the lab and transfer of food from buckets in an outdoor habitat to successfully choose a generous donor in a task intended to assess reputation formation (e.g., Subiaul et al., 2008). Both dogs and cats have recently been shown to choose containers associated with humans eating and showing pieces of food, but both species may exhibit a bias for attending to specific human actions. Despite their different domestication backgrounds and evolutionary histories, both species may learn to attend to these actions because of their shared rearing environment with humans (Chijiiwa et al., 2020). A social lifestyle does not differentiate dogs from cats in a task in which attending to specific physically observable features suffices to allow them to choose the baited container.

On the other hand, applying a rule across completely different tasks or contexts requires more flexibility even if not representing a higher level of abstraction. This kind of generalization can occur within physical and social contexts. For example, following from our earlier example of reputation judgments, if a chimpanzee can infer that an individual that behaves generously in a feeding context might make a better ally in a cooperation task (e.g., Melis et al., 2006), then the chimpanzee may have used information about food sharing to predict performance in a different kind of social task. Of course, the chimpanzee may also simply be choosing an individual for whom it has formed positive regard to interact with in different contexts without reasoning about how their attributes might contribute to performance in various situations. In either case, if the positive regard drives choices across contexts, it allows greater flexibility in an individual's decision making compared with when positive regard is limited to a very specific context - for example, engaging in mutual grooming with one partner consistently while not being tolerant of food sharing with the same individual. This flexibility (or lack thereof) is much easier to measure and quantify, whereas the cognitive representation underlying behavior remains elusive.

The individual that attends to the reliability of an automatic feeder to produce food when pecked may make similar attributions of reliability (reputation) to a nonsocial agent, thus demonstrating the same cognitive process across social and physical tasks. In both cases, the individual is reasoning about reliability of outcome regardless of the specific attributes of the agent. In a physical task, a bird that learns that a preferred food degrades over time (Clayton \& Dickinson, 1999) may also use its understanding of time to predict when berries and fruits may ripen or replenish after longer intervals. Thus far, we know of little 
experimental evidence of such transfer in any species, but animals clearly apply constructs broadly within natural settings. For example, arboreal primates that can learn to use heavier stones (among other features) to crack nuts (Ferreira et al., 2010; Sirianni et al., 2015) presumably also reason that heavier bodies require stronger branches when traversing through the treetops (although even this assumption remains untested). With regard to social behavior, it is almost certain that group-living primates can generalize their understanding that a dominant animal will both displace others at a food source and receive more grooming from their peers (Watts, 2000). Whether this is achieved via a representation of constructs such as dominance or the idea that some individuals reap more benefits than others is difficult to ascertain.

It is even more challenging to present evidence of nonhumans generalizing a rule from a physical context to a social context, or vice versa. Such evidence would help dispel the notion of domain-specific learning. We know of only two relevant empirical studies. Jelbert et al. (2015) examined the transfer of causal cognition from New Caledonian crows using physical tools to using a conspecific as a social tool in a collaborative task and concluded in favor of domain-specific processes. Although the crows could engage in collaborative behavior, their behavior reflected transfer of specific prior experience rather than generalization of a rule regarding the causality of cooperation. Because the use of social tools has been exhibited by species that are both highly social and that use tools, the authors questioned whether the use of social tools has emerged because of highly social lifestyles or a transfer of causal reasoning originating from tool use to the social domain. Based on the performance of the crows in their study, these authors argued for domain specificity in causal cognition, whereas we would argue for a lack of causal understanding more broadly, thus limiting its generalizability. Interestingly, the crows learned to drop stones into an apparatus to release rewards for themselves and a partner, but they had been previously trained to drop stones into a hole, so it is unclear to which extent they understood the causal role of dropping the stone in the current task or if they were simply continuing to do what had previously led to being rewarded. Even more social corvids-rooks-performed similarly in related studies; they were unable to learn to wait for their partner in the loose string cooperative task (Scheid \& Noe, 2010; Seed et al., 2008). Thus, social complexity again did not predict better performance in a task that required reasoning about both conspecifics and a physical apparatus.
In another groundbreaking study involving birds, Bastos and Taylor (2020) showed that kea integrate information across social and physical domains to infer events involving sampling. For example, they use knowledge of relative quantities as well as biases of experimenters and physical constraints, such as barriers, to predict sampling events. The authors take their findings as evidence of domain-general cognition in kea. Another example of a domain-general mechanism that can easily be imagined to apply in both social and physical domains is the capacity to perform transitive inference. In the social domain, an animal that can compute and track dominance rankings can successfully predict which conspecifics would prevail in a conflict even if they have never observed an altercation between those specific conspecifics (Paz-y-Miño et al., 2004). In the physical domain, this ability could be applied to tracking the impact of various time intervals on depleted food resources even when never having foraged after a specific time interval. So, for example, if a scrub jay knows that a mealworm degrades after both $18 \mathrm{-hr}$ and 24-hr intervals, it does not need direct experience to realize that there is no benefit to searching sites $20 \mathrm{hr}$ after caching mealworms there. When tested for the ability to track dyadic relationships, social species appear to outperform less social species (Bond et al., 2003, 2010), but the question of transfer from social to nonsocial contexts has seldom been addressed. The work of Bastos and Taylor and of Bond and colleagues serve as a useful framework for conducting further work with additional species.

Despite the ability to imagine the application of these kinds of cognitive processes across domains, it is difficult to present data that support these domain-general abilities in any species. This is due in part to the reality that researchers typically test animals on one or a small number of specific problems at a time. If test batteries are given, even those designed to test knowledge in both physical and social domains (e.g., Herrmann et al., 2010; MacLean et al., 2017), they do not explicitly test the transfer of newly acquired knowledge across tasks, making it impossible to distinguish between generalization of knowledge and independent learning or innate abilities. Many abilities that give the appearance of complexity are likely sculpted by an animal's evolutionary history and actually provide little evidence of flexibility. The case of the cleaner wrasse described next illustrates this point.

Cleaner wrasses are important members of the coral reef ecosystem, keeping host fish clean of parasites when they arrive in areas known as "cleaning stations." Cleaners will often partake in cheating behaviors when they eat the protective mucus layer from the host fish, rather than con- 
suming the parasites and bacteria, resulting in the host fish punishing the cleaner. To combat this adverse response, cleaners (the agent) swim toward nearby predators, using them as social tools, to protect against such punishments (the target). Wismer and colleagues (2016) found that cleaners discriminated between models of predator and nonpredator species and were subsequently able to generalize this knowledge to novel pairs of models. They were able to accomplish this independent of features such as shape, color, and familiarity. The predator models included morphologically distinct species such as moray eels and groupers, originating from both the cleaners' native Great Barrier Reef and the Caribbean. Although the cleaners had no previous experience with the Caribbean species, they were morphologically and ecologically similar to their Great Barrier Reef counterparts (i.e., the Nassau grouper and the peacock cod). The cleaners' ability to discriminate between predator and nonpredator models of species with which they had no possible previous encounters suggests that they were able to generalize based on previous experiences with comparable species from their native territory; however, it is unknown on what basis the generalization occurred. This ability to effectively discriminate between predator and nonpredator species is necessary for the implementation of a broad "predator is safe haven" rule that the cleaners demonstrated: When faced with a predator and nonpredator species, the predator species will provide security from the attacking host fish, whereas a nonpredator species will provide no such cover.

The "predator is safe haven" rule is ecologically relevant and particularly salient for the cleaners. In contrast, when presented with two models of the same category (i.e., two predator models or two nonpredator models), the cleaners showed no preference for the "correct" model that would provide superior protection, suggesting that they were unable to abstract a "one-of-two stimuli is safe haven" rule, as it lacks ecological validity. From an ecological perspective, neither situation presents a superior choice that would result in better protection from punishment; with two predator models, both would theoretically provide safe haven, whereas with two nonpredator models, neither would provide safe haven. Thus, the ability to discriminate between two models in the same category is not sufficiently salient to the cleaners to develop an effective rule (Wismer et al., 2016). Cleaners' ability to formulate and generalize a rule regarding safe haven from punishment is largely based on their natural ecology and not flexible.

This finding highlights the importance of considering species' natural ecologies when interpreting results as evidence of transfer. Although it provides important context to demonstrate that cleaners did not develop a "one-of-two stimuli is safe haven" rule, this result serves only to confirm that placing too much emphasis on their ability to seemingly abstract and generalize the "predator is safe haven" rule when presented with novel model pairs may lead to an overstatement of their cognitive abilities. To discount their natural ecology would be to suppose more sophisticated cognitive abilities for a behavior that, although seemingly complex, is highly canalized. In an elegant series of studies, Prétôt and colleagues (2016a, 2016b) demonstrated the importance of structuring a task with features relevant to a species' natural ecology before drawing strong conclusions regarding species differences. Cleaner fish surprisingly outperformed primates in a dichotomous choice task, but in this case, because the result was surprising, the researchers probed further to understand why the primates underperformed in this task (Prétôt et al., 2016a). Unfortunately, when results of comparative studies confirm researchers' biases, researchers typically do not engage in this kind of post hoc testing to give the underperforming species the best chance to demonstrate their abilities. Typical experimental tasks are biased toward primates, often using visual or auditory cues rather than tactile or olfactory cues and allowing primates to manipulate objects using hands and fingers, similar to how humans would respond. Researchers have often focused on presenting different species with the same task (often based on tasks first presented to humans) instead of investigating the same ability using different tasks that are uniquely geared toward the study species (see also Eaton et al., 2018; Vonk et al., 2020). These biases are likely to provide confirming evidence for hypotheses emphasizing the importance of social complexity given our own similarity to other social species like primates. These methodological challenges will need to be overcome before we can determine which species truly demonstrate across-context transfer.

\section{Generalizing Across Experiences}

Knowledge can also be applied from one's own experience or perspective to imagining that of another or from another's imagined perspective or experience to one's own. Two of the most influential developmental psychologists-Piaget and Vygotsky-advocated for alternative models of learning, with Piaget focused on direct experience and Vygotsky focused on learning from experts within one's cultural milieu. Piaget's model of intellectual development emphasizes the acquisition of 
knowledge through the individual's direct experience with their environment (Papert, 1967; Piaget, 1967). The individual progresses through a series of stages from a focus on self-directed to outward actions and increasingly abstract representations. Piaget differentiated between social, physical, and logico-mathematical knowledge. Social knowledge is based on social conventions, whereas physical knowledge is based on physical experiences. Logico-mathematical knowledge consists of relationships between objects. In this model, Piaget discussed cognitive functions, which remain constant, and cognitive structures, which change quantitatively and qualitatively over a lifetime (Papert, 1967; Piaget, 1967). Piaget's theory of constructivism lays the groundwork for recognizing the interaction of innate abilities and learning based on specific environmental inputs.

\section{Mother-Offspring Interactions}

One particularly important context for learning occurs from mother to offspring. Species communally reared by their mother, siblings, and other group members, as in African wild dogs (Malcolm \& Marten, 1982) and prairie voles (Solomon, 1991), encourage the transmission of knowledge over generations. Some carnivore mothers (e.g., domestic cats: Caro, 1980; tigers: Schaller, 1967; lions: Schenkel, 1966; cheetahs: Kruuk \& Turner, 1967; otters: Liers, 1951) modify their hunting behavior in a series of stages so that their young can mimic them in a manageable sequence, building more complex behaviors on top of simpler, easier-to-learn behaviors (Leyhausen, 1979). Caro (1994) documented the changes in mother cheetahs' predatory behaviors as their cubs age. When the cubs are younger than 1 month, females kill the prey themselves, but when the cubs are about 5 months old, mothers release their prey in front of their cubs and expect them to kill it, thus providing them with the direct experience of killing prey (Caro, 1994). Similarly, omnivorous meerkat adults assist in the provision of young pups. As the pups grow, the adults kill, disable, or leave the prey (e.g., a scorpion) intact before providing it to the pups (Thornton \& McAuliffe, 2006). It is expected that the offspring generalize from these specific experiences to develop into proficient hunters in their own right. When they produce their own young, mothers will repeat these same "teaching" actions. If the mothers have reenacted behaviors that were directed toward them early in life, they may have understood that the behavior is of particular value for young dependents. More likely, their own teaching behaviors are initiated by specific behavioral cues from the cubs. As cubs age, they may not need to have the behavior modeled or shaped directly but may learn from observing others engaging in behavior such as hunting. Throughout ontogeny, there may be a shift from learning only through direct experiences to being able to learn vicariously through observing others.

\section{Groupmate Interactions}

In contrast to Piaget's constructivist theory, Vygotsky (1978) emphasized the role of culture more strongly than independent learning. He defined learning as "the acquisition of many specialized abilities for thinking about a variety of things" (p. 36), which aligns well with our view that species develop domain-general abilities that allow them to apply this knowledge across a variety of contexts. Vygotsky emphasized that social competition, communication, and cooperation were facilitated through learning and developed for "culturally organized, ... psychological functions" (p. 40). Through social learning, members of groups learn from observing the behavior of conspecifics; the more time individuals spend in a group, the more diverse the experiences. As such, social inheritance increases, which increases the complexity of cultural and cognitive abilities (Cole \& Packer, 2019). Social learning has allowed some species to more effectively dominate and invade environments, encouraging the evolution of other species around them (Tinker et al., 2009). The social learning of these species rapidly pushes forth the evolution of cognitive abilities, driving the biological evolution of other species at a quicker pace. In this evolutionary arms race, predator and prey are coevolving to outsurvive the other (Abrams, 1986).

Because of the ability to innovate novel behaviors and to acquire these behaviors from groupmates, group-living species such as chimpanzees might exhibit behavioral diversity across populations (Whiten, 2017). However, non-group-living species such as orangutans also show some evidence of cultural transmission, supporting our argument that sociality may not be the key predictor of various generalization abilities. For example, orangutans in Borneo and Sumatra exhibit different patterns of leaf use (van Schaik et al., 2003). It is customary for orangutans at one location in Borneo to wipe their face with leaves, but this behavior is absent in other Bornean populations and in Sumatra. Similarly, behaviors including tree-hole use, seed extraction tool use, and the use of leaves such as sponges or scoops vary between and within Southeastern Asia locations (van Schaik et al., 2003). This variation in tool use suggests that, although physically able to exhibit the same behaviors, orangutan groups have different traditions that allow for the transmission of different behaviors. 
In experimental studies of cultural transmission, researchers are better able to precisely determine the mechanism by which behaviors spread in different populations. For example, using two complex tool-use problems, researchers demonstrated cultural transmission between groups and within groups of chimpanzees (Pan troglodytes; Whiten \& van Schaik, 2007). Foraging innovations spread between one troop and into a subsequent troop, providing evidence that behaviors can be culturally transmitted through observation (Whiten et al., 2007). Although there has often been speculation that culture is specific to primates, traditions have also been indicated outside the primate order. For example, a population of humpback whales (Megaptera novaeangliae) living west of Australia migrated into the territory of another population, and before long the resident population adopted the unique vocalizations of the transient population (Noad et al., 2000). Cetaceans are one of the few groups of mammals that appear to learn vocal production (Janik, 2014; although see Mercado \& Perazio, 2021). Fascinating examples of orca behavior also indicate that mother killer whales change their individual vocal signature when pregnant so that their offspring imitate this novel signature. Once the young whale is proficient with the new song, mothers revert to their own signature so that each maintains its own individual call (Weiss et al., 2006). Presumably, the change in a female's song production is mediated by hormonal changes during pregnancy rather than a conscious effort to teach one's young, but either way, it reflects an important and adaptive degree of flexibility.

\section{Interspecific Interactions}

Whereas instances of cultural transmission are usually documented within species, there are rare, documented cases of direct interspecific transmission of behaviors. In at least one species of moths (Asian corn borer, Ostrinia funacalis), male moths mimic their natural bat predator calls in their courtship song; this discourages male competition and encourages females to "freeze" long enough for copulation (Nakano et al., 2013). In another case of vocalization mimicry and transfer, an African elephant (Loxodonta Africana) housed with two Asiatic elephants (Elephas maximus) acquired Asiatic elephant vocalizations (Poole et al., 2005). Cross-fostering experiments with birds and primates have shown that both taxa learn the vocalizations of host species rather than the calls of their own species (Eriksen et al., 2009; Hansen et al., 2010; Masataka \& Fujita, 1989), although other reports suggest that such learning is rather limited (in macaques; see Owren et al., 1993). It is beyond the scope of this article to discuss all the fascinating examples of intra- and interspecies social learning; it suffices to note that the ability to generalize from observing others to applying similar actions, emotions, and vocal communications to one's own actions is widespread.

\section{Human-Animal Interactions}

Because of anthropogenic changes in the environment, the ability of animals to generalize information from humans is of increasing interest. For example, domestic dogs use novel social cues from humans to find food with or without the human in the room (Hare et al., 2002). Dogs select the correct container after a human physically points to it, while standing on the opposite side of the room with another container, and choose the correct container with no human in the room and only a physical marker on the correct container (Hare et al., 2002). This finding again suggests that there is nothing uniquely social about the skill of using points as a cue to find food, as the skill transfers to the use of a physical cue in the absence of any social agent. Like dogs that evolved from highly social wolves, domestic cats-which evolved from asocial ancestors - when presented with a two-object forced choice task use their owner's gaze and vocal signals to identify the correct food bowl (Pongrácz et al., 2019). The abilities of domestic dogs and cats to make inferences about humans indicate that the social-cognitive abilities that enable them to communicate with humans in unique ways are not unique to an evolutionary history of group living but are skills that are likely honed through the domestication process. Furthermore, asocial reptiles also follow gaze (Simpson \& O'Hara, 2019).

Nondomestic species with extensive experience with humans also demonstrate the ability to read human communicative cues, such as commands to engage in imitative behaviors. Jaakkola and colleagues (2010) trained blindfolded dolphins to copy behaviors of another dolphin, demonstrating that the dolphins could form a representation of another's behavior through echolocation or auditory cues and then enact the same behaviors with their own bodies. Dolphins successfully copied vocal and motor behaviors, exhibiting flexibility in imitation. Dolphins have been documented relating their body to a human body and adopting behaviors that seem similar to those of a human (Herman et al., 1999), even when viewing humans on television screens, which requires an additional ability to understand that the television image represents a live human (Herman, 2002). In addition to social imitation and generalization, dolphins imitating the actions of individuals on a television screen with no human 
or conspecific assistance demonstrates that generalization is not restricted to an interactive social context. Although it could be argued that observing social agents on a television is a social context and still involves reasoning about motivations of social agents, it is absent the kind of interactive behaviors and cues present when interacting with live agents. So-called ghost controls are frequently implemented in studies of social learning to examine whether subjects acquire demonstrated behaviors similarly when no social agent is performing the action. Some authors have demonstrated that animals fail to imitate when they do not ascribe intentionality to the agent, and this appears to be the case for both highly social and less social species (bearded dragons, Kis et al., 2015; marmosets, Burkart et al., 2012). Thus, little transfer of learning from social to nonsocial agents can occur in both highly social and less social species.

There are many interesting examples where individuals of various species apply information gleaned from observing others to their own behavior in similar contexts. For example, individuals can take on complementary roles in cooperative tasks, switching roles as the task requires, which suggests that they are able to generalize from observing another's actions to the mechanics required to produce the action themselves (chimpanzees, Povinelli et al., 1992a; although see Péron et al., 2011, for a failure in African grey parrots and Povinelli et al., 1992b, for a failure in rhesus macaques). New Caledonian crows could learn to perform complementary behaviors in a collaborative task but behaved similarly regardless of whether a partner was present or absent or whether rewards were equal, causing the researchers to question their understanding of the task and their ability to transfer causal knowledge from the physical to the social domain (Jelbert et al., 2015). However, organisms can benefit from eavesdropping the interactions of others (Abdai \& Miklósi, 2016), can acknowledge when they need to teach skills to others (as in the case of mothers teaching their young to hunt), and can reference others for information about how to proceed in ambiguous situations through social referencing (even in asocial species like cats; Merola et al., 2015).

\section{Generalizing From One's Own Experience to Another's and Vice Versa}

One of the recent developments in theory of mind research (discussed next) is the advent of experience projection tasks (Heyes, 1998; Karg et al., 2015; Vonk \& Povinelli, 2011) in which individuals project an internal mental experience of their own onto another in the same situation or context. For example, if you and I are facing each other and I turn my computer screen or my phone to face you when showing you an interesting video of animal behavior, I am recognizing that your perspective is different from mine and that you cannot see what I see. I am generalizing my own visual experience of when I can see an object to your experience based on the line of sight from your eyes to the object. Your visual experience also has implications for your knowledge of that object. In experimental tasks, chimpanzees may be given the experience of being unable to see through opaque visors, goggles, or blindfolds that appear identical on the outside and the experience of wearing transparent visors. They can then generalize that others wearing the same visors can see only when wearing the transparent version and that therefore only that individual will be responsive to silent visual gestures (e.g., Vonk \& Povinelli, 2011). Although extremely clever versions of these paradigms have been developed for use only with apes so far (e.g., Karg et al., 2015), results have not revealed any compelling evidence that even apes can succeed in such tasks. Because the nature of the construct to be generalized is not directly observable (see next), this may be one type of generalization that is beyond the grasp of nonhumans.

However, researchers have argued that some species infer others' goals, presumably through generalizing from their own goals in a given context. For example, apes react differently to a human's action based on whether it is intentional or accidental (Call \& Tomasello, 1998), distinguish the goals of another's actions (Buttelmann et al., 2017), and assist others to complete their goals (Hare \& Tomasello, 2004; Warneken \& Tomasello, 2006). These findings mirror the early studies of Premack, who found that the chimpanzee Sarah could choose an image within a sequence of actions that completed the desired outcome of the actor (Premack \& Woodruff, 1978a, 1978b). These studies are heralded as evidence that apes observe other apes (and even humans) and predict outcomes ostensibly based on the attribution of others' goals, demonstrating the ability to generalize from another's experience to their own and vice versa. They are able to observe another conspecific, infer the preferred behavior in that individual's situation, and act on that to either assist or outcompete the conspecific. Being able to apply this skill in both cooperative and competitive situations demonstrates a form of generalizing of the skill across contexts within the social domain. However, even such demonstrations can (and indeed must) be explained by reference to the generalization of observable features or cues as to the outcome without reference to the internal mental state (see the aforemen- 
tioned Asymmetric Dependency problem; Povinelli, 2020; Povinelli \& Vonk, 2004). Here, we are less concerned with the nature of the underlying representation and its abstractness but focus on the extent to which the information can be generalized across experiences and contexts.

\section{Generalizing From Others' Knowledge}

Organisms may also recognize that other individuals have knowledge that they do not have and that may be exploited to serve their own goals. For example, with the concept of social tool use, the subject uses another individual (conspecific or otherwise), rather than a physical object, to reach a goal or target (Bard, 1990; Whiten \& Byrne, 1988). When one individual (the agent) follows another individual (the social tool) to a lucrative foraging patch (the target), the agent capitalizes on the knowledge of the social tool to reach their target without having to expend additional effort to search for a food source. The ability for an individual to generalize rules about the effective implementation of social tools in different contexts suggests a possible propensity toward generalization across experiences. The informed forager paradigm has been applied in pigs (Held et al., 2000), horses (Andrieu et al., 2016), and nonhuman primates (Hall et al., 2017), and it demonstrates that many species can exploit the knowledge of others for their own gains, showing that they generalize what another knows to their own situation.

Some species are also able to draw inferences from third-party interactions. That is, observing two other individuals interacting may produce knowledge that is relevant for the observer. For example, an observer may determine who is dominant between two other individuals, and this will have direct relevance when they must decide who to recruit as an ally or who to compete against for resources. Chimpanzees have been documented "eavesdropping" on other members of the troop to their own benefit (Melis et al., 2006), a behavior that is evident in a vast number of taxa (birds: Johnstone, 2001; Mennill et al., 2002; Otter et al., 1999; fish: McGregor \& Doutrelant, 2000; Oliviera et al., 2001; lemur and sifaka: Fitchel, 2004; Oda \& Masataka, 1996; marmoset and squirrel monkeys: Shriner, 1998; dik-dik: A. J. Lea et al., 2008; hyena, lion, and African wild dog: H. Webster et al., 2010). Chimpanzees infer the reputation of human strangers who behaved either generously or selfishly toward another individual (Subiaul et al., 2008). When given the option to request food from either the selfish or generous stranger, chimpanzees preferred to beg from generous donors that they had not previously interacted with themselves (Subiaul et al., 2008). In a related study, Russell et al. (2008) found that chimpanzees preferred to sit close to generous versus selfish strangers after observing third-party interactions. It might be assumed that sociality contributes to the ability to learn from third-party interactions as less social species (e.g., domestic cats: Chijiiwa et al., 2021; Leete et al., 2020) have not yet shown reputation effects, but the ability has also been elusive in domestic dogs (Kundey et al., 2011; Nitzschner et al., 2012; Piotti et al., 2017). In fact, apparent evidence for reputation effects in dogs may be explained by local enhancement (Jim et al., 2020). Lack of affirming evidence for asocial species may have less to do with being reared in asocial environments than with a lack of skill for transferring information from others' perspectives. In support of this hypothesis, individuals with autism - a developmental disorder with social interaction impairments - have also failed to demonstrate reputation formation (Izuma et al., 2011). Furthermore, Suraci et al. (2017) provided evidence for possible eavesdropping in black bears, which are relatively solitary, and Herrmann et al. (2013) found that both orangutans and chimpanzees approached nice versus mean experimenters after indirect observations. These findings are consistent with our view that the distinction between social and asocial species is less useful for predicting skill sets, social and physical, than presently assumed.

Using information gleaned from observing third-party interactions and applying that to predict one's own interactions can be effective in reducing the cost of experiencing things firsthand. Information obtained through direct experience is more accurate but imposes costs to the individual, whereas social information-information obtained indirectly, through other individuals - is less expensive for the individual but less reliable. According to the "costly information hypothesis," individuals should maximize their personal benefits, relying on social information when personal (directly obtained) information is too costly, and prioritizing personal information when the cost is relatively low (M. M. Webster \& Laland, 2008). In line with this hypothesis, species apply rules flexibly across a variety of contexts to ensure an advantageous cost-benefit ratio. Honeybees, for example, employ a "copy-if-dissatisfied" rule (Gruter et al., 2013). The premise of this rule is predicated on bees' predisposition to rely on previous experience when determining where to forage. As long as the individual bee is satisfied with their energy yield from known food sources, they are less likely to rely on social information from conspecifics. This rule focuses on the benefits more than the cost; if the benefits of relying on personal information decrease such that the individual is no longer 
satisfied with their food source, they will then rely on social information to increase their benefits.

In contrast to the copy-if-dissatisfied rule, other species, such as minnows (M. M. Webster \& Laland, 2008) and guppies (Kendal et al., 2004), exhibit a "copyif-asocial-learning-is-costly" rule, which focuses more on the costs. When perceived predation risk was increased, the reliance on social information subsequently increased in both species. Guppies demonstrated a preference for foraging alone as long as the remainder of their school was in sight; however, when the distance became such that they lost visual contact, the benefits of foraging alone were rapidly outweighed by the potential predation costs that foraging as a group mitigates (Kendal et al., 2004). In a more recent study, however, Hasenjager and Dugatkin (2017) showed an interesting divergence from this established pattern. They found that social information transference was related to present ecological conditions, specifically predation threat. When the threat of predation was low, the guppies exhibited increased transmission of social information, and when the threat was high, there was less evidence of transmission. The researchers suggested that this pattern may result from an overall lack of firsthand information during times of elevated risk, lending to a perceived increase in cost of relying on deficient social information; it may be better to accept the cost of using firsthand information in a situation where there may be an even higher cost to relying on inadequate social information. This suggests that guppies flexibly apply social rules to a variety of situations dependent on the cost to them. Interestingly, social information is applied in a physical context - that of foraging, again blurring the distinction between domains.

Cowbirds also appear to follow this copy-if-asociallearning-is-costly rule, in the contexts of both foraging and nest selection. Cowbirds are brood parasites, often opting to lay their eggs in the nest of an unsuspecting host bird. Females determine the most economical decision, reliance on social or personal information, depending on the context and their present status (Davies \& White, 2018). Successful nest prospecting generally correlates with foraging success in individuals that show a reliance on personal information, whereas those that do not exhibit the same level of individual success should resort to social information to maintain a competitive edge in nest prospecting. In a systematic examination of this pattern of behaviors, Davies and White (2018) found that the degree to which female cowbirds relied on social information was inverse to the amount of personal information they had previously accrued. Researchers found that it was not a lack of attention to social information that dictated individual's use of such information but rather the active decision as to what sources of information they relied upon in different contexts. When they lacked direct information regarding the location of a food source, they demonstrated a proclivity to rely heavily upon social information, suggesting the implementation of context-specific rules that assure maximization of benefits.

It is not solely the sociality of a species that allows for the differential application of rules across a variety of contexts but also the potential for individuals to acquire different levels of personal information. When all individuals in a group have the same information, it negates the advantages of applying context-specific rules about when to rely on directly or socially acquired information. It is only when there is the potential for individuals to have novel, privileged information that they can share or withhold to their greatest benefit that context-specific rules are adaptive. Similarly, it would be adaptive for such rules to develop in species that encounter diversity in the content of both personal and social information. For species with a generalist ecology, the amount and variety of information available to be shared socially is higher than in those with a specialist ecology.

The ability to generalize information based on both direct and indirect social interactions comes from a variety of taxa. Evidence for this type of generalization is most likely ascertained from highly social species because of their natural exposure to intricate social groupings. In particular, we acknowledge that these generalization abilities may be heavily documented in primates because of the surplus of literature and research dedicated to these species. Primates have been studied for decades because of their genetic proximity to humans. Thus, an abundance of evidence for abilities deemed particular to social species cannot be interpreted as such without investigating similar mechanisms in less social species. Shifting our focus from domain-specific to domain-general abilities will illuminate many parallel abilities in less social species as well. Perhaps most telling, the mechanisms of social learning are not unique to social species. For example, social learning has been demonstrated in asocial reptiles such as lizards and turtles (Davis \& Burghardt, 2011; Kis et al., 2015). Mechanisms for learning via observation can be adaptive even for relatively solitary species. Animals need not inhabit social groups to encounter other organisms. In these encounters, learning about another's method of food extraction would be beneficial so long as one has the requisite morphology to benefit from this knowledge. Observing predators preying on other prey species may also 
inform an organism of that predator's status as a potential threat. However, we need not think of these instances of learning from interactions of agents as any different from learning about the interaction of objects through observation, so there is no reason to assume that social learning is specifically social or possible only for social species. In addition to demonstrations that asocial species can learn socially, recently researchers have suggested that individual learning processes may play a stronger role in learning behaviors such as tool use (e.g., Bernstein-Kurtycz et al., 2020; Bandini \& Tennie, 2020) than previously considered. If these researchers are correct, then the adoption of particular strategies or techniques is heavily dependent upon context and available resources and the benefits of social living may have been overstated.

\section{Generalizing Across Observed versus Inferred Experiences}

One of many outstanding questions in comparative psychology is the extent to which nonhumans can reason about unobservable causal forces, which can be applied in both social and physical domains (Vonk \& Povinelli, 2006). Because unobservables cannot, in principle, be directly observed via any sensory modality, they can only be inferred from directly observable events, associations, or behaviors. This sets up the Asymmetric Dependency problem (Povinelli, 2020; Povinelli \& Vonk, 2004) in which a system for representing conceptual and causal relations necessarily depends upon a system that represents observable features and events alone, which makes the latter system necessary and sufficient for explaining most (if not all) cases of transfer that have been championed by researchers as evidence for the former.

The ability to reason about unobservables is presumably facilitated by symbolic representation because words and/or symbols can be used to represent constructs that cannot be directly referenced (Macphail, 1985; Premack, 1983). Although there is still some debate (Lyn, \& Christopher, 2020), it is generally agreed that nonhumans do not exhibit a true symbolic communication system, which should inhibit both their ability to represent unobservables and researchers' ability to uncover such an ability should it exist. However, if it could be demonstrated that nonhumans infer the presence of an underlying causal force from observable events and further generalize the operation of this same force in other circumstances, this would constitute a flexible representation of associations between observable events and behavioral contingencies rather than an inflexible response based on operant reinforcement. For example, an individual that understands a conspecific's knowledge about where food is hidden can apply this knowledge to predict different behaviors such as pattern of search in a foraging situation and aggression against competitors, all motivated by the same knowledge state.

Although difficult to study empirically, one unobservable construct that has received an immense amount of attention from experimenters constitutes the broad array of mental states (e.g., desires, intentions, beliefs, knowledge states, emotions). The ability to represent such states in others is known as theory of mind (ToM; Premack \& Woodruff, 1978b). ToM was long championed as a uniquely human capacity, although recently researchers have begun to consider that some animals, such as great apes, may possess some components of ToM (Buttelmann et al., 2017; Kano et al., 2019; Krupenye et al., 2016). The idea that some components of an attribute such as ToM may be present in lieu of the entire suite of abilities is a novel and important consideration (see also de Waal \& Ferrari, 2010; Smith et al., 2012). Recognizing this possibility will allow researchers to detect greater variability in abilities compared with when they are searching for all-or-none capacities. Acknowledging that components of an attribute may appear to different degrees will increase the likelihood of identifying key factors in the expression of these components by increasing the variability in the trait to be accounted for. Thus far, only highly social animals have demonstrated evidence of some components of ToM, such as perspective-taking. For example, corvids (Bugnyar \& Heinrich, 2006; Bugnyar et al., 2016; Dally et al., 2006; Güntürkün \& Bugnyar, 2016) and canids (Maginnity et al., 2014) have been ascribed with the ability to reason about states of visual attention. Evidence for understanding of second-order false belief states, even in apes (Call \& Tomasello, 2008; Kaminski et al., 2008; Karg et al., 2016; Krachun et al., 2009, 2010), has been more elusive. Furthermore, even the most compelling research has been rigorously evaluated and subject to alternative interpretation (Andrews, 2018; Ben-Yami, 2016; Heyes, 2017; Scarf \& Ruffman, 2017).

Although researchers have claimed to overcome the Asymmetric Dependency problem by devising new tests with greater controls (Krupenye et al., 2016) or different types of rules that must be generalized (Lurz \& Krachun, $2011,2019)$, it is an inescapable reality that any behavioral test must present subjects with observable cues on which they could infer the underlying mental state. Thus, any pattern of results can be explained with reference to the behavioral states alone (Povinelli \& Vonk, 2004). Although 
experience projection tasks (such as the goggles task discussed earlier; Vonk \& Povinelli, 2011) may hold the most promise, even these have been critiqued (Andrews, 2005; Lurz \& Krachun, 2019). Even if the goal of identifying ToM incontrovertibly in nonhumans is unattainable, researchers can determine which species show the ability to generalize across diverse cues, or track more complex interactions, and the extent to which these skills are tied to various aspects of social and foraging complexity.

Although experimental tests are diagnostic only with regard to the type of observable cues that subjects can generalize from, there exists great variability in performance in such tests. Most species fail to reach the level of performance that researchers would accept (erroneously, because of the fundamental logical flaw with such tests) as evidence for ToM. Researchers have sometimes argued that species fail ToM tests because they are being asked to reason about the mental states of humans, rather than of conspecifics (Maginnity \& Grace, 2014). However, the same researchers do not launch this criticism when animals respond to human cues successfully (e.g., Buttelmann et al., 2017; Hare et al., 2006; Kano et al., 2019; Krupenye et al., 2016). Nor do they highlight the fact that humans readily extend their mental state attributions to other species. In fact, ToM is so dominant within human cognition that we find it difficult not to attribute mental states such as intentionality to even inanimate objects. If nonhumans are limited to reasoning about mental states in only their conspecifics, and only in certain contexts, their ToM is significantly limited in comparison (see also Vonk $\&$ Povinelli, 2006). This is a fundamental case of failure to exhibit cognitive flexibility.

Likewise, if chimpanzees can wield ToM only in competitive but not cooperative contexts (Hare, 2001; Hare $\&$ Tomasello, 2004), a severe limitation in generalization is exhibited. Therefore, it is still an open question as to which species might share some components of the ToM capacity present in humans, and the extent to which the ability can be generalized beyond contexts that mirror evolutionary conditions that acted as selection pressures for the trait. Presumably, the adaptive function of ToM is to allow an organism flexibility in responding to the outcome of a particular inner mental state when a novel outward behavior is expressed, thus providing greater predictive power and flexibility than if one did not have ToM. If the employment of ToM yields no greater predictive power than a system that reasons about the observable cues on which inferences of ToM are based, it seems unlikely it would evolve in any species, including humans, especially considering the presumed additional burden it would impose on brain development.
Two outstanding questions remain: Why would humans alone develop ToM? What adaptive benefits does such an ability confer that led to its emergence? A possible advantage of the ability to reason about mental states is to explain events after they have occurred. However, little research has addressed whether other species engage in backward reasoning (Povinelli \& Dunphy Lelii, 2001) despite the importance of this question (Völter et al., 2020). Although it seems reasonable to assume that ToM would evolve in highly social species in order to understand the intentions of groupmates, it is unclear what element of sociality would be most predictive of ToM, and less socially complex species have not been tested in the most promising paradigms. Given that various highly social species have been tested and have not passed tests purported to measure ToM, and nonsocial species have not been tested and therefore not been shown to fail these tests, there is no compelling reason to accept the commonly assumed association between complex sociality and ToM abilities (Dunbar, 2014; Tomasello, 2000).

Furthermore, other predictive factors have seldom been explored. Although researchers have presumed a role of domestication in shaping the social cognition of domestic dogs (Hare \& Tomasello, 2005), dogs were selectively bred from wolves that exhibit a high degree of sociality and a somewhat generalist diet. Therefore, it would be difficult to disentangle the role of an evolutionary history of complex social behavior, foraging complexity, and domestication in shaping canine cognition. Comparisons of the abilities of domestic dogs and wolves suggest that wolves often perform similarly to domestic dogs in tests of social and physical cognition, particularly when they have been exposed to humans from an early age (Brucks et al., 2019; Heberlein et al., 2016; Range \& Virányi, 2013). Such studies are complicated by the fact that nonhumans are often tested for their understanding of human mental states rather than for their understanding of conspecifics' mental states, and the subjects' history with humans is not always adequately accounted for (see Marshall-Pescini et al., 2017, for a review of the need to account for the species' social ecology). Udell and Wynne (2010) articulated a two-stage domestication hypothesis in which the subjects' ontogenetic history was as important as the domestication process in determining its ability to read human communicative states, harkening back to our earlier point about considering the individual's history in addition to the ecology of its species.

Although it is more intuitive to suppose that development of a system for reading mental states would depend upon the need to assess motives, attention, and knowledge 
in close associates, it is possible that perspective taking emerged as a broader need to assess the attention of potential prey or predators rather than conspecifics. Alternatively, ToM may have emerged from a more general cognitive capacity for the representation of multiple possible physical states, which might be beneficial for species that engage in extractive foraging or track the temporal and spatial availability of food and other resources. Thus far, researchers have focused only on aspects of canid ecology that relate to sociality. Dietary factors have not been explicitly linked to the general capacity to represent multiple states in mind simultaneously - the basic foundation for ToM capacities (but see S. E. G. Lea \& Osthaus, 2018).

Of course, mental states are only one special class of unobservables. Other causal forces include gravity, transfer of force, and other physical forces. Whereas one can observe the outward manifestations of such forces, one cannot observe the force itself. As with most hypotheticals, there is evidence that humans reason about such constructs because of our ability to communicate such ideas in speech and writing. When animals evidence some understanding of such principles, it is difficult if not impossible to disentangle their understanding from reliance on the perceptual cues that are correlated with the force itself. And reasoning about the physical cues alone, because of this almost perfect correspondence, is just as predictive, at least within highly similar contexts (Vonk \& Povinelli, 2006). Therefore, this type of transfer may not only be the most difficult for nonhumans to achieve, it is also the most challenging to obtain evidence for. Note that we are not assuming that such abilities do not exist, just that there is little available evidence to draw on. It is also premature to conclude whether social or foraging complexity contribute to such abilities, given the absence of clear evidence in nonhuman species.

\section{General Conclusions}

To date, researchers have been distracted by attempts to firmly categorize cognitive mechanisms underlying interesting behaviors into associative or cognitive strategies while failing to acknowledge the futility of such distinctions. We argue that it will be more productive to dispense with such fixations and examine the flexibility with which animals apply knowledge and skills across varied tasks and situations. Although we cannot use transfer performance to assess generalization of the most complex cognitive representations that we know humans can master, existing examples of generalizations continue to reveal interesting patterns of performance that indicate the breadth of transfer of knowledge and experience. This variability in transfer across features, contexts, and experiences can help researchers determine the contributions of various aspects of sociality and foraging complexity to shaping cognition in a wide range of species.

Given the paucity of similar tests across animals exhibiting less complex sociality and foraging strategies, it is premature to draw conclusions regarding the selection pressures responsible for cognitive flexibility. However, we suspect that the need to flexibly adapt to changing environments (Sol, 2009a, 2009b) will stand out as an important predictor. We have identified several other challenges in identifying factors responsible for shaping cognition. First, researchers have exhibited dichotomous thinking when parsing animals into social versus asocial categories rather than representing the true complexity of animals' social environments. Reproductive and parenting strategies and interactions between predators and prey have not featured heavily in the discussion of cognitive evolution. Although examination of foraging strategies has identified patchy food distribution and the need for extractive foraging as important factors in shaping cognition (Byrne, 2012; Milton, 1981), animals have also been classified as generalists versus specialists with regard to foraging strategies, and a full picture of how these factors interact to predict cognition has not yet emerged. Perhaps the most striking omission is the lack of theories incorporating both sociality and foraging strategies as interacting predictors. Cognition has not emerged in a vacuum, so it is critical that researchers attend to multiple predictive factors rather than ocusing on single predictors in isolation.

Thus far, it is not clear whether more highly social species exhibit greater cognitive flexibility compared with less highly social species. The latter species have provided far less data, particularly with regard to social cognitive tasks, making it difficult to determine associations with particular aspects of foraging complexity independently of social factors. However, in the vast evidence we have reviewed for various types of generalization, we see no reason to suppose that sociality confers any specific advantage either specifically within the social domain or more broadly. The question of whether any advantages conferred by environmental factors are domain specific or domain general has not been advanced despite its importance.

Enough information has accrued to allow us to conclude that humans are unique in both abstraction and breadth of generalization abilities. Perhaps it is the unique confluence of social and physical factors (i.e., the need to rely on social connections in their unique agricultural economy) that has allowed for uniquely human cognition. 
We see much overlap between social and physical cognitive skills and suggest that a blurring of the distinction between domains and a focus on seeking domain-general transference of knowledge and skill will yield more informative insights for the study of cognitive evolution. We prefer to think of social and physical domains, not as two distinct domains each requiring their own unique cognitive processes but as different contexts in which the same cognitive processes can be flexibly applied.

\section{References}

Abdai, J., \& Miklósi, Á. (2016). The origin of social evaluation, social eavesdropping, reputation formation, image scoring or what you will. Frontiers in Psychology, 7, Article 13. https://doi.org/10.3389/ fpsyg.2016.01772

Abrams, P. A. (1986). Is predatory-prey coevolution an arms race? Trends in Ecology \& Evolution, 1(4), 108110. https://doi.org/10.1016/0169-5347(86)90037-6

Allman, J., McLaughlin, T., \& Hakeem, A. (1993). Brain weight and life-span in primate species. Proceedings of the National Academy of Sciences, 90(1), 118-122. https://doi.org/10.1073/pnas.90.1.118

Amodio, P., Jelbert, S. A., \& Clayton, N. S. (2018). The interplay between psychological predispositions and skill learning in the evolution of tool use. Current Opinion in Behavioral Sciences, 20, 130-137. https:// doi.org/10.1016/j.cobeha.2018.01.002

Andrews, K. (2005). Chimpanzee theory of mind: Looking in all the wrong places? Mind \& Language, 20(5), 521536. https://doi.org/10.1111/j.0268-1064.2005.00298.x

Andrews, K. (2018). Apes track false beliefs but might not understand them. Learning \& Behavior, 46(1), 3-4. https://doi.org/10.3758/s13420-017-0288-8

Andrieu, J., Henry, S., Hausberger, M., \& Thierry, B. (2016). Informed horses are influential in group movements, but they may avoid leading. Animal Cognition, 19(3), 451-458. https://doi.org/10.1007/s10071-015-0945-2

Arden, R., \& Adams, M. J. (2016). A general intelligence factor in dogs. Intelligence, 55, 79-85. https://doi. org/10.1016/j.intell.2016.01.008

Audet, J., \& Lefebvre, L. (2017). What's flexible in behavioral flexibility? Behavioral Ecology, 28(4), 943-947. https://doi.org/10.1093/beheco/arx007
Bandini, E., \& Tennie, C. (2020). Exploring the role of individual learning in animal tool use. PeerJ, 8, e9877. https://doi.org/10.7717/peerj.9877

Bard, K. A. (1990). Social tool use by free-ranging orangutans: A Piagetian and developmental perspective on the manipulations of an inanimate object. In S. T. Parker \& K. R. Gibson (Eds.), "Language” and intelligence in monkeys and apes: Comparative developmental perspectives ( $\mathrm{pp}$. 356-378). Cambridge University Press. https://doi.org/10.1017/ CBO9780511665486.015

Bar-Hen-Schweiger, M., \& Henik, A. (2020). The transition of object to mental manipulation: Beyond a species-specific view of intelligence. Animal Cognition, 23(4), 691-701. https://doi.org/10.1007/s10071-020$01375-2$

Barker, K. B., \& Povinelli, D. J. (2019) Anthropomorphomania and the rise of the animal mind: A conversation. Journal of Folklore Research, 56(1-2), 71-90. https://doi.org/10.2979/jfolkrese.56.2_3.05

Bastos, A. P. M., \& Taylor, A. H. (2020). Kea show three signatures of domain-general statistical inference. Nature Communications, 11, Article 828. https://doi.org/10.1038/s41467-020-14695-1

Bentley-Condit, V. K., \& Smith, E. O. (2009). Animal tool use: Current definitions and an updated comprehensive catalog. Behaviour, 147, 185-221. https://doi.org/10.11 63/000579509X12512865686555

Ben-Yami, H. (2016, October 21). Apes still don't understand much [Online commentary]. Science. https://science.sciencemag.org/content/354/6308/110/ tab-e-letters.

Bernstein-Kurtycz, L. M., Hopper, L. M., Ross, S. R., \& Tennie, C. (2020). Zoo-housed chimpanzees can spontaneously use tool sets but perseverate on previously successful tool-use methods. Animal Behavior and Cognition, 7(3), 288-309. https://doi.org/10.26451/ abc.07.03.03.2020

Bird, C. D., \& Emery, N. J. (2009). Insightful problem solving and creative tool modification by captive nontool-using rooks. Proceedings of the National Academy of Sciences, 106, 10370-10375. https://doi. org/10.1073/pnas.0901008106 
Boesch, C. (2003). Complex cooperation among taï chimpanzees. In F. B. M. de Waal \& P. L. Tyack (Eds.), Animal social complexity: Intelligence, culture, and individualized societies (pp. 93-110). Harvard University Press. https://doi.org/10.4159/ harvard.9780674419131.c7

Boesch, C. (2020). Mothers, environment, and ontogeny affect cognition. Animal Behavior and Cognition, 7(3), 474-489. https://doi.org/10.26451/abc.07.03.13.2020

Bond, A. B., Kamil, A. C., \& Balda, R. P. (2003). Social complexity and transitive inference in corvids. Animal Behaviour, 65(3), 479-487. https://doi.org/10.1006/ anbe.2003.2101

Bond, A. B., Wei, C. A., \& Kamil, A. C. (2010). Cognitive representation in transitive inference: A comparison of four corvid species. Behavioural Processes, 85(3), 283-292. https://doi.org/10.1016/j.beproc.2010.08.003

Boucherie, P. H., Loretto, M., Massen, J. J. M., \& Bugnyar, T. (2019). What constitutes "social complexity" and "social intelligence" in birds? Lessons from ravens. Behavioral Ecology and Sociobiology, 73, Article 14. https://doi.org/10.1007/s00265-018-2607-2

Boyd, R., Richerson, P. J., \& Henrich, J. (2011). The cultural niche: Why social learning is essential for human adaptation. Proceedings of the National Academy of Sciences of the United States of America, 108(Suppl. 2), 10918-10925. https://doi.org/10.1073/ pnas. 1100290108

Brajon, S., Laforest, J., Bergeron, R., Tallet, C., \& Devillers, N. (2015). The perception of humans by piglets: Recognition of familiar handlers and generalizations to unfamiliar humans. Animal Cognition, 18, 1299-1316. https://doi.org/10.1007/s10071-015-0900-2

Brucks, D., Marshall-Pescini, S., \& Range, F. (2019). Dogs and wolves do not differ in their inhibitory control abilities in a non-social test battery. Animal Cognition, 22(1), 1-15. https://doi.org/10.1007/s10071-018-12169

Bugnyar, T., \& Heinrich, B. (2006). Pilfering ravens, Corvus corax, adjust their behavior to social context and identity or competitors. Animal Cognition, 9(4), 369-376. https://doi.org/10.1007/s10071-006-0035-6
Bugnyar, T., Reber, S. A., \& Buckner, C. (2016). Ravens attribute visual access to unseen competitors. Nature Communications, 7, Article $10506 . \mathrm{https} / /$ doi. org/10.1038/ncomms 10506

Burkart, J., Kupferberg, A., Glasauer, S., \& van Schaik, C. (2012). Even simple forms of social learning rely on intention attribution in marmoset monkeys (callithrix jacchus). Journal of Comparative Psychology, 126(2), 129-138. https://doi.org/10.1037/a0026025

Burkart, J. M., Schubiger, M. N., \& van Schaik, C. P. (2017). The evolution of general intelligence. Behavioral and Brain Sciences, 40, Article e195. https://doi.org/10.1017/S0140525X16000959

Buttelmann, D., Buttelmann, F., Carpenter, M., Call, J., \& Tomasello, M. (2017). Great apes distinguish true from false beliefs in an interactive helping task. PLOS ONE, 12(4), Article e0173793. https://doi.org/10.1371/ journal.pone. 0173793

Buttelmann, D., Schütte, S., Carpenter, M., Call, J., \& Tomasello, M. (2012). Great apes infer others' goals based on context. Animal Cognition, 15, 1037-1053. https://doi.org/10.1007/s10071-012-0528-4

Byrne, R. (1997). The technical intelligence hypothesis: An additional evolutionary stimulus to intelligence? In A. Whiten \& R. Byrne (Eds.), Machiavellian intelligence II: Extensions and evaluations (pp. 289-311). Cambridge University Press. https://doi. org/10.1017/CBO9780511525636.012

Byrne, R., \& Whiten, A. (Eds.). (1988). Machiavellian intelligence: Social expertise and the evolution of intellect in monkeys, apes and humans I. Oxford Science Publications.

Call, J., \& Tomasello, M. (1998). Distinguishing intentional from accidental actions in orangutans (pongo pygmaeus), chimpanzees (pan troglodytes) and human children (homo sapiens). Journal of Comparative Psychology, 112(2), 192-206. https://doi. org/10.1037/0735-7036.112.2.192

Call, J. \& Tomasello, M. (2008). Does the chimpanzee have a theory of mind? 30 years later. Trends in Cognitive Sciences, 12(5), 187-192. https://doi.org/10.1016/j. tics.2008.02.010 
Caro, T. M. (1980). Predatory behaviour in domestic cat mothers. Behaviour, 74(1-2), 128-147. https://doi. org $/ 10.1163 / 156853980 X 00357$

Caro, T. (1994). Cheetahs of the Serengeti Plains: Group living in an asocial species. University of Chicago Press.

Castles, M., Heinsohn, R., Marshall, H. H., Lee, A. E. G., Cowlishaw, G., \& Carter, A. J. (2014). Social networks created with different techniques are not comparable. Animal Behaviour, 96, 59-67. https://doi. org/10.1016/j.anbehav.2014.07.023

Chance, M. R. A. (1960). Kohler's chimpanzees: How did they perform? Man, 60, 130-135. https://doi. org/10.2307/2797042

Chijiiwa, H., Takagi, S., Arahori, M., Anderson, J. R., Fujita, K., \& Kuroshima, H. (2021). Cats (Felis catus) show no avoidance of people who behave negatively to their owner. Animal Behavior and Cognition, 8(1), 23-35. https://doi.org/10.26451/abc.08.01.03.2021

Chijiiwa, H., Takagi, S., Arahori, M., Hori, Y., Saito, A., Kuroshima, H., \& Fujita, K. (2020). Dogs and cats prioritize human action: Choosing a now-empty instead of a still-baited container. Animal Cognition, 24, 65-73. https://doi.org/10.1007/s10071-020-01416-w

Clayton, N. S., \& Dickinson, A. (1999). Scrub jays (aphelocoma coerulescens) remember the relative time of caching as well as the location and content of their caches. Journal of Comparative Psychology, 113(4), 403-416. https://doi.org/10.1037/0735-7036.113.4.403

Clayton, N. S., \& Emery, N. J. (2007). The social life of corvids. Current Biology, 17, R652. https://doi. org/10.1016/j.cub.2007.05.070

Cole, M.,\&Packer,M.(2019).Culture and cognition.InK.D. Keith (Ed.), Cross-cultural psychology: Contemporary themes and perspectives (2nd ed.; pp. 243-270). Wiley \& Sons. https://doi.org/10.1002/9781119519348.ch11

Colombo, M., Scarf, D., \& Zentall, T. (2021). Editorial: The comparative psychology of intelligence: Macphail's revisted. Frontiers in Psychology, 12, Article 648782. https://doi.org/10.3389/fpsyg.2021.648782

Dally, J. M., Emery, N. J., \& Clayton, N. S. (2006). Food-caching western scrub-jays keep track of who was watching when. Science, 312(5780), 1662-1665. https://doi.org/10.1126/science.1126539
Damerius, L. A., Burkart, J. M., van Noordwijk, M. A., Haun, D. B. M., Kosonen, Z. K., Galdikas, B. M. F., . . . van Schaik, C. P. (2019). General cognitive abilities in orangutans (pongo abelii and pongo pygmaeus). Intelligence, 74, 3-11. https://doi. org/10.1016/j.intell.2018.10.008

Davies, H. B., \& White, D. J. (2018). Specializations in cognition generalize across contexts: Cowbirds are consistent in nest prospecting and foraging tasks. Animal Behaviour, 144, 1-7. https://doi.org/10.1016/j. anbehav.2018.07.019

Davis, K. M., \& Burghardt, G. M. (2011). Turtles (pseudemys nelsoni) learn about visual cues indicating food from experienced turtles. Journal of Comparative Psychology, 125(4), 404-410. https://doi.org/10.1037/ a0024784

Dean, L. G., Kendal, R. L., Schapiro, S. J., Thierry, B. \& Laland, K. N. (2012). Identification of the social and cognitive processes underlying human cumulative culture. Science 335(6072), 1114-1118. https://doi. org/10.1126/science.1213969

Deaner, R. O., Barton, R. A., \& Schaik, C. P. v. (2003). Primate brains and life histories: Renewing the connection. In P. M. Kappeler \& M. E. Pereira (Eds.), Primate life histories and socioecology; primate life histories and socioecology (pp. 233-265). University of Chicago Press.

Deecke, V. B. (2012). Tool-use in the brown bear (Ursus arctos). Animal Cognition, 15(4), 725-730. https://doi. org/10.1007/s10071-012-0475-0

DeTroy, S. E., Ross, C. T., Cronin, K. A., van Leeuwen, E. J. C., \& Haun, D. B. M. (2021). Cofeeding tolerance in chimpanzees depends on group composition: A longitudinal study across four communities. ISCIENCE. https://doi.org/10.1016/j.isci.2021.102175

de Waal, F. (2016). Are we smart enough to know how smart animals are? W. W. Norton \& Company.

de Waal, F. B. M., \& Ferrari, P. F. (2010). Towards a bottom-up perspective on animal and human cognition. Trends in Cognitive Sciences, 14(5), 201-207. https:// doi.org/10.1016/j.tics.2010.03.003 
Diez, L., Moquet, L., \& Detrain, C. (2013). Post-mortem changes in chemical profile and their influence on corpse removal in ants. Journal of Chemical Ecology, 39, 1424-1432. https://doi.org/10.1007/ s10886-013-0365-1

Dunbar, R. I. M. (1998). The social brain hypothesis. Evolutionary Anthropologist, 6, 178-190. https://doi. org/10.1002/(SICI)1520-6505(1998)6:5<178::AIDEVAN5>3.0.CO;2-8

Dunbar, R. I. M. (2014). What's so social about the social brain? In J. Decety \& Y. Christen (Eds.), New frontiers in social neuroscience (pp. 1-10). Springer International Publishing. https://doi.org/10.1007/9783-319-02904-7 1

Dunbar, R. I. M., \& Shultz, S. (2007). Evolution in the social brain. Science, 317(5843), 1344-1347. https:// doi.org/10.1126/science. 1145463

Eaton, T., Hutton, R., Leete, J., Lieb, J., Robeson, A. \& Vonk, J. (2018). Bottoms-up: Rejecting top-down human-centered approaches in comparative psychology. International Journal of Comparative Psychology, 31. https://doi.org/10.46867/ijcp.2018.31.01.11

Elbroch, M. L., Levy, M., Lubell, M., Quigley, H., \& Caragiulo, A. (2017). Adaptive social strategies in a solitary carnivore. Science Advances, 3(10), Article e1701218. https://doi.org/10.1126/sciadv.1701218

Emery, N. J., \& Clayton, N. S. (2009). Tool use and physical cognition in birds and mammals. Current Opinion in Neurobiology, 19, 27-33. https://doi.org/10.1016/j. conb.2009.02.003

Epstein, R. (1981). On pigeons and people: A preliminary look at the Columbian simulation project. Behavioral Analysis, 4,43-55.https://doi.org/10.1007/BF03391851

Epstein, R., Kirshnit, C. E., Lanza, R. P., \& Rubin, L. C. (1984). 'Insight' in the pigeon: Antecedents and determinants of an intelligent performance. Nature, 308, 61-62. https://doi.org/10.1038/308061a0

Eriksen, A., Lampe, H. M., \& Slagsvold, T. (2009). Interspecific cross-fostering affects song acquisition but not mate choice in pied flycatchers ficedula hypoleuca. Animal Behaviour, 78(4), 857-863. https:// doi.org/10.1016/j.anbehav.2009.07.005
Ferreira, R. G., Emidio, R. A., \& Jerusalinsky, L. (2010). Three stones for three seeds: Natural occurrence of selective tool use by capuchins (cebus libidinosus) based on an analysis of the weight of stones found at nutting sites. American Journal of Primatology, 72(3), 270-275. https://doi.org/10.1002/ajp.20771

Freeberg, T. M., Dunbar, R. I., \& Ord, T. J. (2012). Social complexity as a proximate and ultimate factor in communicative complexity. Philosophical Transactions of the Royal Society B: Biological Sciences, 367, 1785-1801. https://doi.org/10.1098/rstb.2011.0213

Fichtel, C. (2004). Reciprocal recognition of sifaka (Propithecus verreauxi verreauxi) and redfronted lemur (eulemur fulvus rufus) alarm calls. Animal Cognition, 7(1), 45-52. https://doi.org/10.1007/s10071-003-01800

Fuller, A. R. (1990). Insight into value. State University of New York Press.

Gavrilets, S., \& Vose, A. (2006). The dynamics of Machiavellian intelligence. Proceedings of the National Academy of Sciences, 103(45), 16823-16828. https:// doi.org/10.1073/pnas.0601428103

Gittleman, J. L. (1986). Carnivore brain size, behavioral ecology, and phylogeny. Journal of Mammalogy, 67, 23-36. https://doi.org/10.2307/1380998

Goodall, J. (1964). Tool-Using and aimed throwing in a community of free-living chimpanzees. Nature, 201, 1264-1266. https://doi. org/10.1038/2011264a0

Gruter, C., Segers, F., \& Ratnieks, F. L. W. (2013). Social learning strategies in honeybee foragers: Do the costs of using private information affect the use of social information? Animal Behaviour, 85, 1443-1449. https:// doi.org/10.1016/j.anbehav.2013.03.041

Güntürkün, O., \& Bugnyar, T. (2016). Cognition without cortex. Trends in Cognitive Sciences, 20(4), 291-303. https://doi.org/10.1016/j.tics.2016.02.001

Hall, K., Oram, M. W., Campbell, M. W., Eppley, T. M., Byrne, R. W., \& de Waal, F. B. M. (2017). Chimpanzee uses manipulative gaze cues to conceal and reveal information to foraging competitor. American Journal of Primatology, 79(3), 1-11. https://doi.org/10.1002/ ajp.22622 
Hansen, B. T., Johannessen, L. E., \& Slagsvold, T. (2010). Interspecific cross-fostering of great tits (parus major) by blue tits (cyanistes caeruleus) affects inter- and intraspecific communication. Behaviour, 147(3), 413-424. https://doi.org/10.1163/00057950 9X12578482434926

Hare, B. (2001). Can competitive paradigms increase the validity of experiments on primate social cognition? Animal Cognition, 4(3-4), 269-280. https://doi. org/10.1007/s100710100084

Hare, B., Brown, M., Williamson, C., \& Tomasello, M. (2002). The domestication of social cognition in dogs. Science, 298(5598), 495-514. https://doi.org/10.1126/ science. 1072702

Hare, B., Call, J., Agnetta, B., \& Tomasello, M. (2000). Chimpanzees know what conspecifics do and do not see. Animal Behaviour, 59(4), 771-785. https://doi. org/10.1006/anbe.1999.1377

Hare, B., Call, J., \& Tomasello, M. (2006). Chimpanzees deceive a human competitor by hiding. Cognition, 101(3), 495-514. https://doi. org/10.1016/j.cognition.2005.01.011

Hare, B., \& Tomasello, M. (2004). Chimpanzees are more skillful in competitive than cooperative cognitive tasks. Animal Behaviour, 68(3), 571-581. https://doi. org/10.1016/j.anbehav.2003.11.011

Hare, B., \& Tomasello, M. (2005). Human-like social skills in dogs? Trends in Cognitive Sciences, 9(9), 439-444. https://doi.org/10.1016/j.tics.2005.07.003

Harrison, M. E., \& Chivers, D. J. (2007). The orang-utan mating system and the unflanged male: A product of increased food stress during the late Miocene and Pliocene? Journal of Human Evolution, 52, 275-293. https://doi.org/10.1016/j.jhevol.2006.09.005

Hart, B. L., Hart, L. A., McCoy, M., \& Sarath, C. R. (2001). Cognitive behaviour in Asian elephants: Use and modification of branches for fly switching. Animal Behaviour, 62, 839-847. https://doi.org/10.1006/ anbe. 2001.1815

Hasenjager, M. J., \& Dugatkin, L. A. (2017). Familiarity affects network structure and information flow in guppy (poecilia reticulata) shoals. Behavioral Ecology, 28(1), 233-242. https://doi.org/10.1093/beheco/arw152
Hauser, M. D. (1988). Invention and social transmission: New data from wild vervet monkeys. In R. Byrne \& A. Whiten (Eds.), Machiavellian intelligence: Social expertise and the evolution of intellect in monkeys, apes and man I (pp. 327-343). Oxford Science Publications.

Heberlein, M. T. E., Turner, D. C., Range, F., \& Virányi, Z. (2016). A comparison between wolves, Canis lupus, and dogs, Canis familiaris, in showing behaviour towards humans. Animal Behaviour, 122, 59-66. https://doi. org/10.1016/j.anbehav.2016.09.023

Held, S., Mendl, M., Devereux, C., \& Byrne, R. W. (2000). Social tactics of pigs in a competitive foraging task: The “informed forager" paradigm. Animal Behaviour, 59(3), 569-576. https://doi.org/10.1006/anbe.1999.1322

Herman, L. M. (2002). Vocal, social, and self-imitation by bottlenose dolphins. In K. Dautenhahn \& C. L. Nehaniv (Eds.), Imitation in animals and artifacts (pp. 63-108). MIT Press.

Herman, L. M., Abichandani, S. L., Elhajj, A. N., Herman, E. Y. K., Sanchez, J. L., \& Pack, A. A. (1999). Dolphins (Tursiops truncatus) comprehend the referential character of the human pointing gesture. Journal of Comparative Psychology, 113(4), 347-364. https://doi. org/10.1037/0735-7036.113.4.347

Hernik, M., \& Csibra, G. (2009) Functional understanding facilitates learning about tools in human children. Current Opinion in Neurobiology, 19(1), 34-38. https:// doi.org/10.1016/j.conb.2009.05.003

Herrmann, E., Hernández-Lloreda, M. V., Call, J., Hare, B., \& Tomasello, M. (2010). The structure of individual differences in the cognitive abilities of children and chimpanzees. Psychological Science, 21(1), 102-110. https://doi.org/10.1177/0956797609356511

Herrmann, E., Keupp, S., Hare, B., Vaish, A., \& Tomasello, M. (2013). Direct and indirect reputation formation in nonhuman great apes (pan paniscus, pan troglodytes, gorilla gorilla, pongo pygmaeus) and human children (homo sapiens). Journal of Comparative Psychology, 127(1), 63-75. https://doi.org/10.1037/ a0028929

Heyes, C. M. (1998). Theory of mind in nonhuman primates. Behavioral and Brain Sciences, 21(1), 101134. https://doi.org/10.1017/S0140525X98000703 
Heyes, C. (2017). Apes submentalise. Trends in Cognitive Sciences, 21(1), 1-2. https://doi.org/10.1016/j. tics.2016.11.006

Holzhaider, J. C., Sibley, M. D., Taylor, A. H., Singh, P. J., Gray, R. D., \& Hunt, G. R. (2011). The social structure of new Caledonian crows. Animal Behaviour, 81(1), 83-92. https://doi.org/10.1016/j.anbehav.2010.09.015

Humphrey, N. K. (1976). The social function of intellect. In P. P. G. Bateson \& R. A. Hinde (Eds.), Growing points in ethology (pp. 303-317). Cambridge University Press.

Iwaniuk, A. N., Wylie, D. R., \& Lefebvre, L. (2009). The comparative approach and brain-behaviour relationships: A tool for understanding tool use. Canadian Journal of Experimental Psychology, 63, 150-159. https://doi.org/10.1037/a0015678

Izuma, K., Matsumoto, K., Camerer, C. F., \& Adolphs, R. (2011). Insensitivity to social reputation in autism. Proceedings of the National Academy of Sciences, 108(42), 17302-17307. https://doi.org/10.1073/ pnas. 1107038108

Jaakkola, K., Guarino, E., \& Rodriguez, M. (2010). Blindfolded imitation in a bottlenose dolphin (Tursiops truncatus). International Journal of Comparative Psychology, 23(4), 671-688. https://doi. org/10.5070P4234020475

Janik, V. M. (2014). Cetacean vocal learning and communication. Current Opinion in Neurobiology, 28, 60-65. https://doi.org/10.1016/j.conb.2014.06.010

Jelbert, S. A., Singh, P. J., Gray, R. D., \& Taylor, A. H. (2015). New Caledonian crows rapidly solve a collaborative problem without cooperative cognition. PLoS ONE, 10(8), Article e0133253. https://doi. org/10.1371/journal.pone.0133253

Jim, H-L., Marshall-Pescini, S., \& Range, F. (2020) Do dogs eavesdrop on human interactions in a helping situation? PLOS ONE, 15(8), Article e0237373. https:// doi.org/10.1371/journal.pone. 0237373

Johnson-Ulrich, L., Johnson-Ulrich, Z., \& Holekamp, K. (2018). Proactive behavior, but not inhibitory control, predicts repeated innovation by spotted hyenas tested with a multi-access box. Animal Cognition, 21(3), 379-392. https://doi.org/10.1007/s10071-018-1174-2
Johnstone, R. A. (2001). Eavesdropping and animal conflict. Proceedings of the National Academy of Sciences, 98(16), 9177-9180. https://doi.org/10.1073/ pnas. 161058798

Jolly, A. (1966). Lemur social behaviour and primate intelligence. Science, 153, 501-506. https://doi. org/10.1126/science.153.3735.501

Kamel, S. J., \& Mrosovsky, N. (2005). Repeatability of nesting preferences in the hawksbill sea turtle, Eretmochelys imbricate, and their fitness consequences. Animal Behaviour, 70(4), 819-828. https://doi. org/10.1016/j.anbehav.2005.01.006

Kaminski, J., Call, J., \& Tomasello, M. (2008). Chimpanzees know what others know, but not what they believe. Cognition, 109(2), 224-234. https://doi. org/10.1016/j.cognition.2008.08.010

Kano, F., Krupenye, C., Hirata, S., Tomonaga, M., \& Call, J. (2019). Great apes use self-experience to anticipate an agent's action in a false-belief test. PNAS Proceedings of the National Academy of Sciences of the United States of America, 116(42), 20904-10909. https://doi. org/10.1073/pnas.1910095116

Kappeler, P. M., Clutton-Brock, T., Shultz, S., \& Lukas, D. (2019). Social complexity: Patterns, processes, and evolution. Behavioral Ecology and Sociobiology, 73(5). https://doi.org/10.1007/s00265-018-2613-4

Karg, K., Schmelz, M., Call, J., \& Tomasello, M. (2015). The goggles experiment: Can chimpanzees use selfexperience to infer what a competitor can see? Animal Behaviour, 105, 211-221. https://doi.org/10.1016/j. anbehav.2015.04.028

Karg, K., Schmelz, M., Call, J., \& Tomasello, M. (2016). Differing views: Can chimpanzees do level two perspective-taking? Animal Cognition, 19(3), 555-564. https://doi.org/10.1007/s10071-016-0956-7

Kendal, R. L., Coolen, I., \& Laland, K. N. (2004). The role of conformity in foraging when personal and social information conflict. Behavioral Ecology, 15, 269-177. https://doi.org/10.1093/beheco/arh008

Kis, A., Huber, L., \& Wilkinson, A. (2015). Social learning by imitation in a reptile (pogona vitticeps). Animal Cognition, 18(1), 325-331. https://doi.org/10.1007/ s10071-014-0803-7 
Köhler, W. (2019). The mentality of apes (E. Winter, Trans.). Taylor \& Francis. (Original work published 1925). https://doi.org/10.4324/9781351294966

Krachun, C., Carpenter, M., Call, J., \& Tomasello, M. (2009). A competitive nonverbal false belief task for children and apes. Developmental Science, 12(4), 521535. https://doi.org/10.1111/j.1467-7687.2008.00793.x

Krachun, C., Carpenter, M., Call, J., \& Tomasello, M. (2010). A new change-of-contents false belief test: Children and chimpanzees compared. International Journal of Comparative Psychology, 23(2), 145-165.

Krupenye, C., Kano, F., Hirata, S., Call, J., \& Tomasello, M. (2016). Great apes anticipate that other individuals will act according to false beliefs. Science, 354(6308), 110-113. https://doi.org/10.1126/science.aaf8110

Kruuk, H. \& Turner, M. (1967). Comparative notes on predation by lion, leopard, cheetah, and wild dog in the Serengeti area, East Africa. Mammalia, 31(1), 1-27. https://doi.org/10.1515/mamm.1967.31.1.1

Kundey, S. M. A., De Los Reyes, A., Royer, E., Molina, S., Monnier, B., German, R., \& Coshun, A. (2011). Reputation-like inference in domestic dogs (canis familiaris). Animal Cognition, 14(2), 291-302. https:// doi.org/10.1007/s10071-010-0362-5

Lea, A. J., Barrera, J. P., Tom, L. M., \& Blumstein, D. T. (2008). Heterospecific eavesdropping in a nonsocial species. Behavioral Ecology, 19(5), 1041-1046. https:// doi.org/10.1093/beheco/arn064

Lea, S. E. G., \& Osthaus, B. (2018). In what sense are dogs special? Canine cognition in comparative context. Learning \& Behavior, 46(4), 335-363. https://doi. org/10.3758/s13420-018-0349-7

Leete, J. A., Vonk, J., Oriani, S., Eaton, T., \& Lieb, J. (2020). Domestic cats (Felis silvestris catus) do not infer reputation in humans after direct and indirect experience. Human-Animal Interaction Bulletin, 8, $35-53$.

Lefebvre, L., Micolakakis, N., \& Boire, D. (2002). Tools and brains in birds. Behaviour, 139, 939-973. https:// doi.org/10.1163/156853902320387918

Lefebvre, L., Whittle, P., Lascaris, E., \& Finkelstein, A. (1997). Feeeding innovations and forebrain size in birds. Animal Behaviour, 53(3), 549-560. https://doi. org/10.1006/anbe.1996.0330
Leslie, J. C. (2018). Testing the limits of behavioral analysis: A review of Frans de Waal's are we smart enough to know how smart animals are? Journal of the Experimental Analysis of Behavior, 110(3), 569-585. https://doi.org/10.1002/jeab.482

Leyhausen, P. (1979). Cat behavior: The predatory and social behavior of domestic and wild cats. Garland STPM Press.

Liers, E. E. (1951). Notes on the river otter (Lutra canadensis). Journal of Mammalogy, 32(1), 1-9. https:// doi.org/10.2307/1375406

Lott, D. F. (1991). American bison socioecology. Applied Animal Behaviour Science, 29(1-4), 135-145. https:// doi.org/10.1016/0168-1591(91)90242-P

Lurz, R. W., \& Krachun, C. (2011). How could we know whether nonhuman primates understand others' internal goals and intentions? Solving Povinelli's problem. Review of Philosophy and Psychology, 2(3), 449-481. https://doi.org/10.1007/s13164-011-0068-x

Lurz, R., \& Krachun, C. (2019). Experienceprojection methods in theory-of-mind research: Their limits and strengths. Current Directions in Psychological Science, 28(5), 456-462. https://doi. org/10.1177/0963721419850156

Lurz, R., Krachun, C., Mahovetz, L., Wilson, M. J. G., \& Hopkins, W. (2018). Chimpanzees gesture to humans in mirrors: Using reflection to dissociate seeing from line of gaze. Animal Behaviour, 135, 239-249. https://doi. org/10.1016/j.anbehav.2017.11.014

Lyn, H., \& Christopher, J. L. (2020). How environment can reveal semantic capacities in nonhuman animals. Animal Behavior and Cognition, 7(2), 159-167. https://doi. org/10.26451/abc.07.02.10.2020

MacLean, E. L., Herrmann, E., Suchindran, S., \& Hare, B. (2017). Individual differences in cooperative communicative skills are more similar between dogs and humans than chimpanzees. Animal Behaviour, 126, 41-51. https://doi.org/10.1016/j.anbehav.2017.01.005

Macphail, E. (1985). Vertebrate intelligence: The null hypothesis. Philosophical Transactions of the Royal Society B: Biological Sciences, 308, 37-51. https://doi. org/10.1098/rstb.1985.0008 
Macphail, E. M. (1987). The comparative psychology of intelligence. Behavioral Brain Sciences, 10, 645-695. https://doi.org/10.1017/S0140525X00054984

Maginnity, M. E. \& Grace, R. C. (2014). Visual perspective taking by dogs (Canis familiaris) in a Guesser-Knower task: Evidence for a canine theory of mind? Animal Cognition, 17(6), 1375-1392. https://doi.org/10.1007/ s10071-014-0773-9

Malcolm, J. R. \& Marten, K. (1982). Natural selection and the communal rearing of pups in African wild dogs (Lycaon pictus). Behavioral Ecology and Sociobiology, 10(1), 1-13. https://doi.org/10.1007/BF00296390

Marshall-Pescini, S., Cafazzo, S., Virányi, Z., \& Range, F. (2017). Integrating social ecology in explanations of wolf-dog behavioral differences. Current Opinion in Behavioral Sciences, 16, 80-86. https://doi. org/10.1016/j.cobeha.2017.05.002

Martin-Ordas, G., Call, J., \& Colmenares, F. (2008). Tubes, tables and traps: Great apes solve two functionally equivalent trap tasks but show no evidence of transfer across tasks. Animal Cognition, 11(3), 423-430. https:// doi.org/10.1007/s 10071-007-0132-1

Masataka, N., \& Fujita, K. (1989). Vocal learning of Japanese and rhesus monkeys. Behaviour, 109(3-4), 191-199. https://doi.org/10.1163/156853989X00222

Mazur, R., \& Seher, V. (2008). Socially learned foraging behaviour in wild black bears ursus americanus. Animal Behaviour, 75(4), 1503-1508. https://doi.org/10.1016/j. anbehav.2007.10.027

McGregor, P. \& Doutrelant, C. (2000). Eavesdropping and mate choice in female fighting fish. Behaviour, 137(12), 1655-1668. https://doi.org/10.1163/156853900502763

Mercado III, E., \& Perazio, C. E. (2021). Similarities in composition and transformations of songs by humpback whales (megaptera novaeangliae) over time and space. Journal of Comparative Psychology, 135(1), 28-50. https://doi.org/10.1037/com0000268

Megginson, L. C. (1963). Lessons from Europe for American business. Southwestern Science Quarterly, $44,3-13$.

Melis, A. P., Hare, B., \& Tomasello, M. (2006). Chimpanzees recruit the best collaborators. Science, 311(5765), 1297-1300. https://doi.org/10.1126/ science. 1123007
Mennill, D. J., Ratcliffe, L. M., \& Boag, P. T. (2002). Female eavesdropping on male song contests in songbirds. Science, 296(5569), 873. https://doi. org/10.1126/science.296.5569.873

Merola, I., Lazzaroni, M., Marshall-Pescini, S., \& PratoPrevide, E. (2015). Social referencing and cat-human communication. Animal Cognition, 18(3), 639-648. https://doi.org/10.1007/s10071-014-0832-2

Milton, K. (1981). Distribution patterns of tropical plant foods as an evolutionary stimulus to primate mental development. American Anthropologist, 83(3), 534548. https://doi.org/10.1525/aa.1981.83.3.02a00020

Nakano, R., Takanashi, T., Surlykke, A., Skals, N., \& Ishikawa, Y. (2013). Evolution of deceptive true courtship songs in moths. Scientific Reports, 3, 2003. https://doi.org/10.1038/srep02003

Nitzschner, M., Melis, A. P., Kaminski, J., \& Tomasello, M. (2012). Dogs (canis familiaris) evaluate humans on the basis of direct experiences only. PLoS ONE, 7(10), 9. https://doi.org/10.1371/journal.pone.0046880

Noad, M. J., Cato, D. H., Bryden, M. M., Jenner, M., \& Jenner, K. C. (2000). Cultural revolution in whale songs. Nature, 408(6812), 537. https://doi. org/10.1038/35046199

Oda, R., \& Masataka, N. (1996). Interspecific responses or ringtailed lemurs to playback of antipredator alarm calls given by Verreaux's sifakas. Ethology, 102(3), 441-453. https://doi.org/10.1111/j.1439-0310.1996. tb01138.x

O’Donoghue, E. M., Broschard, M. B., \& Wasserman, E. A. (2020). Pigeons exhibit flexibility but not rule formation in dimensional learning, stimulus generalization, and task switching. Journal of Experimental Psychology: Animal Learning and Cognition, 46(2), 107-123. https://doi.org/10.1037/xan0000234

Oliveria, R. F., Lopes, M., Carneiro, L. A., \& Canário, A. V. (2001). Watching fights raises fish hormone levels. Nature, 409(6819), Article 475. https://doi. org/10.1038/35054128

Osiurak, F., \& Reynaud, E.(2020). The elephant in the room: What matters cognitively in cumulative technological culture. Behavioral and Brain Sciences, 43, Article e156. https://doi.org/10.1017/S0140525X20000291 
Otter, K., McGregor, P. K., Terry, A. M. R., Burford, F. R., Peake, T. M., \& Dabelsteen, T. (1999). Do female great tits (Parus major) assess males by eavesdropping? A field study using interactive song playback. Proceedings of the Royal Society of London, B, Biological Sciences, 266(1426), 1305-1309. https://doi.org/10.1098/ rspb.1999.0779

Owren, M. J., Dieter, J. A., Seyfarth, R. M., \& Cheney, D. L. (1993). Vocalizations of rhesus (macaca mulatta) and Japanese (fuscata) macaques cross-fostered between species show evidence of only limited modification. Developmental Psychobiology, 26(7), 389-406. https://doi.org/10.1002/dev.420260703

Papert, S. (1967). Epistemology of cybernetics, and remarks on finality. In J. Piaget (Ed.), Logic and scientific knowledge. Gallimard.

Patterson, E. M., \& Mann, J. (2011). The ecological conditions that favor tool use and innovation in wild bottlenose dolphins. PLOS ONE, 6(8), Article e22243. https://doi.org/10.1371/journal.pone.0022243

Paz-y-Miño, C., G., Bond, A. B., Kamil, A. C., \& Balda, R. P. (2004). Pinyon jays use transitive inference to predict social dominance. Nature, 430(7001), 778-781. https://doi.org/10.1038/nature02723

Perdue, B., Snyder, R., Zhihe, Z., Marr, J., \& Maple, T. (2011). Sex differences in spatial ability: A test of the range size hypothesis in order carnivore. Animal Behaviour, 7, 380-383. https://doi.org/10.1098/ rsbl.2010.1116

Péron, F., Rat-Fischer, L., Lalot, M., Nagle, L., \& Bovet, D. (2011). Cooperative problem solving in African grey parrots (psittacus erithacus). Animal Cognition, 14(4), 545-553. https://doi.org/10.1007/s10071-011-0389-2

Piaget, J. (Ed.). (1967). Logic and scientific knowledge. Gallimard.

Piotti, P., Spooner, R. M., Jim, H., \& Kaminski, J. (2017). Who to ask for help? Do dogs form an opinion on humans based on skilfulness? Applied Animal Behaviour Science, 195, 93-102. https://doi.org/10.1016/j. applanim.2017.05.024

Pongrácz, P., Szapu, J. S., \& Faragó, T. (2019). Cats (Felis silvestris casus) read human gaze for referential information. Intelligence, 74, 43-52. https://doi. org/10.1016/j.intell.2018.11.001
Poole, J. H., Tyack, P. L., Stoeger-Horwath, A. S., \& Watwood, S. (2005). Elephants are capable of vocal learning. Nature, 434(7032), 455-456. https://doi. org/10.1038/434455a

Povinelli, D. J. (2012). World without weight: Perspectives on an alien mind. Oxford University Press. https://doi. org/10.1093/acprof:oso/9780198570967.001.0001

Povinelli, D. J. (2020). Can comparative psychology crack its toughest nut? Animal Behavior and Cognition, 7(4), 589-652. https://doi.org/10.26451/abc.07.04.09.2020

Povinelli, D. J. \& Dunphy-Lelii, S. (2001). Do chimpanzees seek explanations? Preliminary comparative investigations. Canadian Journal of Experimental Psychology/Revue Canadienne de Psychologie Expérimentale, 55(2), 185-193. https:// doi.org/10.1037/h0087365

Povinelli, D. J., \& Frey, S. H. (2016). Constraints on the exploitation of the functional properties of objects in expert tool-using chimpanzees (pan troglodytes). Cortex: A Journal Devoted to the Study of the Nervous System and Behavior, 82, 11-23. https:// doi.org/10.1016/j.cortex.2016.05.011

Povinelli, D. J., \& Henley, T. (2020). More rope tricks reveal why more task variants will never lead to strong inferences about higher-order causal reasoning in chimpanzees. Animal Behavior and Cognition, 7(3), 392-418. https://doi.org/10.26451/abc.07.03.08.2020

Povinelli, D. J., Nelson, K. E., \& Boysen, S. T. (1992a). Comprehension of role reversal in chimpanzees: Evidence of empathy? Animal Behaviour, 43(4), 633640. https://doi.org/10.1016/S0003-3472(05)81022-X

Povinelli, D. J., Parks, K. A., \& Novak, M. A. (1992b). Role reversal by rhesus monkeys, but no evidence of empathy. Animal Behaviour, 44(2), 269-281. https:// doi.org/10.1016/0003-3472(92)90033-6

Povinelli, D. J., \& Vonk, J. (2004). We don't need a microscope to explore the chimpanzee's mind. Jointly published in Mind and Language, 19, 1-28 and in S. Hurley \& M. Nudds (Eds.), Rational animals (2006). Oxford University Press. https://doi.org/10.1111/j.14680017.2004.00244.x

Premack, D. (1983). The codes of man and beasts. Behavioral and Brain Sciences, 6(1), 125-167. https:// doi.org/10.1017/S0140525X00015077 
Premack, D.,\&Woodruff, G.(1978a). Chimpanzeeproblemsolving: A test for comprehension. Science, 202(4367), 532-535. https://doi.org/10.1126/science.705342

Premack, D., \& Woodruff, G. (1978b). Does the chimpanzee have a theory of mind? Behavioral and Brain Sciences, 1(4), 515-526. https://doi.org/10.1017/ S0140525X00076512

Prétôt, L., Bshary, R., \& Brosnan, S. F. (2016a). Comparing species decisions in a dichotomous choice task: Adjusting task parameters improves performance in monkeys. Animal Cognition, 19(4), 819-834. https:// doi.org/10.1007/s 10071-016-0981-6

Prétôt, L., Bshary, R., \& Brosnan, S. F. (2016b). Factors influencing the different performance of fish and primates on a dichotomous choice task. Animal Behaviour, 119, 189-199. https://doi.org/10.1016/j. anbehav.2016.06.023

Pruetz, J. D., \& Bertolani, P. (2007). Savanna chimpanzees, Pan troglodytes verus, hunt with tools. Current Biology, 17, 412-417. https://doi.org/10.1016/j.cub.2006.12.042

Range, F., \& Virányi, Z. (2013). Social learning from humans or conspecifics: Differences and similarities between wolves and dogs. Frontiers in Psychology, 4, Article 10. https://doi.org/10.3389/fpsyg.2013.00868

Reader, S. M., \& Laland, K. N. (2002). Social intelligence, innovation, and enhanced brain size in primates. Proceedings of the National Academy of Sciences, 99(7), 4436-4441. https://doi.org/10.1073/pnas.062041299

Reader, S. M., \& MacDonald, K. (2003). Environmental variability and primate behavioural flexibility. In S. M. Reader \& K. N. Laland (Eds.), Animal innovation (pp. 83-116). Oxford University Press. https://doi. org/10.1093/acprof:oso/9780198526223.003.0004

Rosenzweig, M. R., \& Bennett, E. L. (1996). Psychobiology of plasticity: Effects of training and experience on brain and behavior. Behavioural Brain Research, 81(1), 57-65. https://doi.org/10.1016/0166-4328(95)00216-2

Russell, Y. I., Call, J., \& Dunbar, R. I. (2008). Image scoring in great apes. Behavioural Processes, 78(1), 108-111. https://doi.org/10.1016/j.beproc.2007.10.009
Sayol, F., Maspons, J., Lapiedra, O., Iwaniuk, A. N., Székely, T., \& Sol, D. (2016). Environmental variation and the evolution of large brains in birds. Nature Communications, 7, Article 13971. https://doi. org/10.1038/ncomms13971

Scarf, D., \& Ruffman, T. (2017). Great apes'insight into the mind: How great? [Online commentary]. Science. https://doi.org/10.1126/science.aaf8110

Schaller, G. B. (1967). The deer and the tiger: A study of wildlife in India. The University of Chicago Press.

Scheid, C., \& Noe, R. (2010). The performance of rooks in a cooperative task depends on their temperament. Animal Cognition, 13, 545-553.https://doi.org/10.1007/ s10071-009-0305-1

Schenkel, R. (1966). Play, exploration, and territoriality in the wild lion. In P. Jewell \& C. Loizos (Eds.), Play, exploration, and territoriality in mammals [Symposium]. Zoological Society of London, London, UK.

Schrauf, A. M., \& Call, J. (2011). Great apes use weight as a cue to find hidden food. American Journal of Primatology, 73, 323-334. https://doi.org/10.1002/ ajp.20899

Shultz, S., \& Dunbar, R. I. (2007). The evolution of the social brain: Anthropoid primates contrast with other vertebrates. Proceedings of the Royal Society Biology, 274(1624), 2429-2436. https://doi.org/10.1098/ rspb.2007.0693

Seed, A. M., \& Byrne, R. (2010). Animal tool-use. Current Biology, 20(23), 1032-1039. https://doi.org/10.1016/j. cub.2010.09.042

Seed, A. M., Clayton, N. S., \& Emery, N. J. (2008). Cooperative problem solving in rooks (Corvus frugilegus). Proceedings of the Royal Society B: Biological Sciences, 275, 1421-1429. https://doi. org/10.1098/rspb.2008.0111

Shettleworth, S. J. (2010). Clever animals and killjoy explanations in comparative psychology. Trends in Cognitive Sciences, 14(11), 477-481. https://doi. org/10.1016/j.tics.2010.07.002

Shriner, W. M. (1998). Yellow-bellied marmot and goldenmantled ground squirrel responses to heterospecific alarm calls. Animal Behaviour, 55(3), 529-536. https:// doi.org/10.1006/anbe.1997.0623 
Simpson, J., \& O'Hara, S. J. (2019). Gaze following in an asocial reptile (eublepharis macularius). Animal Cognition, 22(2), 145-152. https://doi.org/10.1007/ s10071-018-1230-y

Sirianni, G., Mundry, R., \& Boesch, C. (2015). When to choose which tool: Multidimensional and conditional selection of nut-cracking hammers in wild chimpanzees. Animal Behaviour, 100, 152-165. https:// doi.org/10.1016/j.anbehav.2014.11.022

Smith, J. D., Coutinho, M. V. C., Boomer, J., \& Beran, M. J. (2012). Metacognition across species. In J. Vonk \& T. K. Shackelford (Eds.), The Oxford handbook of comparative evolutionary psychology (pp. 274-291). Oxford University Press. https://doi.org/10.1093/ oxfordhb/9780199738182.013.0015

Sol, D. (2009a). The cognitive buffer hypothesis for the evolution of large brains. Cognitive Ecology II, pp. 111-136. https://doi.org/10.7208/ chicago/9780226169378.003.0007

Sol, D. (2009b). Revisiting the cognitive buffer hypothesis for the evolution of large brains. Biology Letters, 5(1), 130-133. https://doi.org/10.1098/rsbl.2008.0621

Solomon, N. G. (1991). Current indirect fitness benefits associated with philopatry in juvenile prairie voles. Behavioral Ecology and Sociobiology, 29(4), 277-282. https://doi.org/10.1007/BF00163985

Spaulding, B., \& Hauser, M. D. (2005). What experience is required for acquiring tool competence? Experiments with two callitrichids. Animal Behaviour, 71, 517-526. https://doi.org/10.1016/j.anbehav.2004.11.024

Striedter, G. F. (2013). Bird brains and tool use: Beyond instrumental conditioning. Brain, Behavior, and Evolution, 82, 55-67. https://doi. org $/ 10.1159 / 000352003$

Strübin, C., Steinegger, M., \& Bshary, R. (2011). On group living and collaborative hunting in the yellow saddle goatfish (parupeneus cyclostomus). Ethology, 117(11), 961-969. https://doi.org/10.1111/j.14390310.2011.01966.x

Subiaul, F., Vonk, J., Barth, J., \&. Okamoto-Barth, S. (2008). Do Chimpanzees learn reputation by observation? Evidence from direct and indirect experience with generous and selfish strangers. Animal Cognition, 11, 611-623. https://doi.org/10.1007/s10071-008-0151-6
Suraci, J. P., Clinchy, M., Roberts, D. J., \& Zanette, L. Y. (2017). Eavesdropping in solitary large carnivores: Black bears advance and vocalize toward cougar playbacks. Ethology, 123(9), 593-599. https://doi. org/10.1111/eth.12631

Tarou, L. R. (2004). An examination of associative learning and spatial memory in foraging of two species of bear (family: Ursidae) (Ailuropoda melanoleuca, Tremarctos ornatus). Dissertation Abstracts International: Section B: The Sciences and Engineering, 64, Article 5260.

Taylor, A. H. (2014). Corvid cognition. WIREs Cognitive Science, 5, 361-372. https://doi.org/10.1002/wcs.1286

Tebbich, S., Seed, A. M., Emery, N. J., \& Clayton, N. S. (2007). Non-tool-using rooks, Corvus frugilegus, solve the trap-tube problem. Animal Cognition, 10, 225-231. https://doi.org/10.1007/s10071-006-0061-4

Tebbich, S., Taborsky, M., Fessl, B., \& Dvorak, M. (2002). The ecology of tool use in the woodpecker finch (Cactospiza pallida). Ecology Letters, 5, 656-664. https://doi.org/10.1046/j.1461-0248.2002.00370.x

Templeton, J. J., Kamil, A. C., \& Balda, R. P. (1999). Sociality and social learning in two species of corvids: The pinyon jay (Gymnorhinus cyanocephalus) and the Clark's nutcracker (Nucifraga Columbiana). Journal of Comparative Psychology, 113(4), 450-455. https://doi. org/10.1037/0735-7036.113.4.450

Tennie, C., Call, J., \& Tomasello, M. (2009) Ratcheting up the ratchet: On the evolution of cumulative culture. Philosophical Transactions of the Royal Society of London B 364, 2405-15. https://doi.org/10.1098/ rstb.2009.0052

Teschke, I., Cartmill, E. A., Stankewitz, S., \& Tebbich, S. (2011). Sometimes tool use is not the key: No evidence for cognitive adaptive specializations in tool-using woodpecker finches. Animal Behaviour, 82, 945-956. https://doi.org/10.1016/j.anbehav.2011.07.032

Teschke, I., \& Tebbich, S. (2011). Physical cognition and tool-use: Performance of Darwin's finches in the twotrap tube task. Animal Cognition, 14, 555-563. https:// doi.org/10.1007/s10071-011-0390-9 
Teschke, I., Wascher, C. A., Scriba, M. F., von Bayern, A. M., Huml, V., Siemers, B., \& Tebbich, S. (2013). Did tool-use evolve with enhanced physical cognitive abilities? Philosophical Transactions of the Royal Society B: Biological Sciences, 368, Article 20120418. https://doi.org/10.1098/rstb.2012.0418

Thornton, A., \& McAuliffe, K. (2006). Teaching in wild meerkats. Science, 313(5784), 227-229. https://doi. org/10.1126/science. 1128727

Tinker, M. T., Mangel, M., \& Estes, J. A. (2009). Learning to be different: Acquired skills, social learning, frequency dependence, and environmental variation can cause behaviourally mediated foraging specializations. Evolutionary Ecology Research, 11(6), 841-869.

Tomasello, M. (1999). The human adaptation for culture. Annual Review of Anthropology, 28, 509-529. https:// doi.org/10.1146/annurev.anthro.28.1.509

Tomasello, M. (2000). Two hypotheses about primate cognition. In C. Heyes \& L. Huber (Eds.), The evolution of cognition (pp. 165-183). MIT Press.

Tomasello, M., \& Call, J. (1997). Primate cognition. Oxford University Press.

Udell, M. A., \& Wynne, C. D. L. (2010). Ontogeny and phylogeny: Both and essential to human-sensitive behavior in the genus canis. Animal Behaviour, 79(2), e9-e14. https://doi.org/10.1016/j.anbehav.2009.11.033

van Horik, J. O., \& Emery, N. J. (2016). Transfer of physical understanding in a non-tool-using parrot. Animal Cognition, 19, 1195-1203. https://doi.org/10.1007/ s10071-016-1031-0

van Schaik, C. P., Ancrenaz, M., Borgen, G., Galdikas, B., Knott, C.D., Singleton, I., Suzuki, A., Suci, S., \& Merrill, M. (2003). Orangutan cultures and the evolution of material culture. Science, 299(5603), 102-105. https:// doi.org/10.1126/science.1078004

Visalberghi, E., Sabbatini, G., Taylor, A. H., \& Hunt, G. R. (2017) Cognitive insights from tool use in nonhuman animals. In J. Call (Ed.), APA handbook of comparative psychology, Vol. 2: Perception, learning and cognition (pp. 673-701). American Psychological Association. https://doi.org/10.1037/0000012-030
Visalberghi, E., \& Trinca, L. (1989). Tool use in the capuchin monkeys: Distinguishing between performing and understanding. Primates, 30, 511-521. https://doi. org/10.1007/BF02380877

Völter, C. J., Lambert, M. L., \& Huber, L. (2020). Do nonhumans seek explanations? Animal Behavior and Cognition, 7(3), 445-451. https://doi.org/10.26451/ abc.07.03.10.2020

von Bayern, A. M. P., Heathcote, R. J. P., Rutz, C., \& Kacelnik. (2009). The role of experience in problem solving and innovative tool use in crows. Current Biology, 19, 1965-1968. https://doi.org/10.1016/j. cub.2009.10.037

Vonk, J. (2018). Social tolerance in not-so-social pumas. Learning and Behavior, 46, 105-106. https://doi. org/10.3758/s13420-017-0312-z

Vonk, J. (2019). A fish-eye view of the mirror test. Learning and Behavior, 48, 193-194. https://doi.org/10.3758/ s13420-019-00385-6

Vonk, J. (in press). What laboratory and field approaches bring to bear for understanding the evolution of ursid cognition. In M. Papini, M. Krause, \& K. Hollis (Eds.), The evolution of learning and memory mechanisms. Cambridge University Press.

Vonk, J., \& Edge, J. (in press-a). Do monkeys belong in the ape house? Comparing cognition across primate species. In B. Schwartz \& M. J. Beran (Eds.), Primate cognitive studies. Cambridge.

Vonk, J., \& Edge, J. (in press-b). False dichotomies in the study of animal cognition. In S. Della Sala (Ed.), Encyclopedia of behavioural neuroscience 2nd edition. Elsevier. https://doi.org/10.1016/B978-0-12-809324$5.23955-2$

Vonk, J., Edge, J., Pappas, J., Robeson, A., \& Jordan, A. (2020). Cross species comparisons: When comparing apples to oranges is fruitful. In T. K. Shackelford (Ed.), The Sage handbook of evolutionary psychology (pp. 285310). Sage. https://doi.org/10.4135/9781529739442. n16 
Vonk, J., \& Povinelli, D. J. (2006). Similarity and difference in the conceptual systems of primates: The unobservable hypothesis. In E. Wasserman \& T. Zentall (Eds.), Comparative cognition: Experimental explorations of animal intelligence (pp. 363-387). Oxford University Press. https://doi.org/10.1093/ acprof:oso/9780195377804.003.0020

Vonk, J., \& Povinelli, D. J. (2011). Preliminary investigations of cognitive plasticity: Social and physical causality in home-reared chimpanzees. In N. Eilan, H. Lerman, \& J. Roessler (Eds.), Perception, causation, and objectivity (pp. 342-367). Oxford University Press.

Vygotsky, L. (1978). Interaction between learning and development. Readings on the Development of Children, 23(3), 34-41.

Warneken, F., Chen, F., \& Tomasello, M. (2006). Cooperative activities in young children and chimpanzees. Child Development, 77(3), 640-663. https://doi.org/10.1111/j.1467-8624.2006.00895.x

Warneken, F., \& Tomasello, M. (2006). Altruistic helping in human infants and young chimpanzees. Science, 311(5765), 1301-1303. https:// doi.org/10.1126/science.1121448

Waroff, A. J., Fanucchi, L., Robbins, C. T., \& Nelson, O. L. (2017). Tool use, problem-solving, and the display of stereotypic behaviors in the brown bear (Ursus arctos). Journal of Veterinary Behavior: Clinical Applications and Research, 17, 62-68. https://doi.org/10.1016/j. jveb.2016.11.003

Watts, D. P. (2000). Grooming between male chimpanzees at Ngogo, Kibale national park. II. influence of male rank and possible competition for partners. International Journal of Primatology, 21(2), 211-238. https://doi. org/10.1023/A:1005421419749

Webster, H., McNutt, J. W., \& McComb, K. (2010). Eavesdropping and risk assessment between lions, spotted hyenas, and African wild dogs. Ethology, 116(3), 233-239. https://doi.org/10.1111/j.14390310.2009.01729.x

Webster, M. M., \& Laland, K. N. (2008). Social learning strategies and predation risk: Minnows copy only when using private information would be costly. Proceedings of the Royal Society B., 275, 2869-2876. https://doi. org/10.1098/rspb.2008.0817
Weiss, B. M., Ladich, F., Spong, P., \& Symonds, H. (2006). Vocal behavior of resident killer whale matrilines with newborn calves: The role of family signatures. The Journal of the Acoustical Society of America, 119(1), 627-635. https://doi.org/10.1121/1.2130934

Whiten, A. (2017). Social learning and culture in child and chimpanzee. Annual Review of Psychology, 68, 129-154. https://doi.org/10.1146/annurev-psych-010416-044108

Whiten, A., \& Byrne, R. W. (1988). Tactical deception in primates. Behavioral Brain Science, 11, 233-244. https://doi.org/10.1017/S0140525X00049682

Whiten, A., \& van Schaik, C. P. (2007). The evolution of animal "cultures" and social intelligence. Philosophical Transactions of the Royal Society B: Biological Sciences, 362(1480), 603-620. https://doi.org/10.1098/ rstb.2006.1998

Wismer, S., Grutter, A., \& Bshary, R. (2016). Generalized rule application in bluestreak cleaner wrasse (Labroides dimidiatus): Using predator species as social tools to reduce punishment. Animal Cognition, 19, 769-778. https://doi.org/10.1007/s10071-016-0975-4 